\section{Tobacco use among the elderly: a systematic review and meta-analysis}

\author{
Uso de tabaco entre idosos: revisão sistemática \\ e meta-análise
}

\author{
${ }^{1}$ Instituto de Psiquiatria, \\ Universidade Federal do Rio \\ de Janeiro, Rio de Janeiro, \\ Brasil. \\ 2 GlaxoSmithKline do Brasil, \\ Rio de Janeiro, Brasil. \\ 3 Faculdade de Ciências \\ Médicas, Universidade do \\ Estado do Rio de Janeiro, \\ Rio de Janeiro, Brasil. \\ ${ }^{4}$ Escola Nacional de Saúde \\ Pública Sergio Arouca, \\ Fundação Oswaldo Cruz, \\ Rio de Janeiro, Brasil. \\ 5 Departamento de \\ Psiquiatria, Universidade \\ Federal de São Paulo \\ São Paulo, Brasil. \\ Correspondence \\ V. Marinho \\ Instituto de Psiquiatria, \\ Universidade Federal do \\ Rio de Janeiro. \\ Rua Bento Lisboa 40 \\ apto. 601, Rio de Janeiro, $R J$ \\ 22221-011, Brasil. \\ vmarinho@rionet.com.br
}

Valeska Marinho 1,2

Jerson Laks 1,3

Evandro Silva Freire Coutinho 4

Sergio Luís Blay ${ }^{5}$

\section{Introduction}

The aim of this study was to combine the results of identified surveys on the prevalence of tobacco use in old age to estimate world prevalence of tobacco use and possible factors related to such behavior among the elderly. The literature search included electronic databases such as MEDLINE, LILACS, and Biological Abstracts, hand-searching of specialist journals and cited reference searches. The combined global prevalence was estimated using the random effects model. The total number of elderly subjects included in all surveys was 140,058, with data available from all the continents. Overall prevalence of tobacco use was $13 \%$ in both genders $(22 \%$ male and $8 \%$ female). The prevalence rates were heterogeneous among surveys and were associated with smoking definition, questionnaire application, and country economic status. Few epidemiological studies assessed tobacco use among the elderly. A higher prevalence rate of tobacco use in males who live in higher income countries could be found, although additional evidence regarding elderly samples is still required.

Tobacco; Smoking; Aged
Tobacco use is responsible for almost 10,000 deaths each day and approximately 4.9 million deaths per year worldwide 1 . Cigarette smoking has been linked to several diseases such as respiratory and ischemic heart diseases, stroke, lung, upper respiratory and digestive tract cancers, and peptic ulcer 1 . This habit may contribute to the morbidity and disability associated with many of those illnesses, and it results in a burden of US\$ 157 billion in health-related economic costs in the United States 2.

Smoking is now recognized as a major public health problem also among the elderly. Risk of death among older smokers is higher than among their nonsmoking counterparts. Morbidity and mortality from cancer, stroke, cardiovascular, and respiratory diseases are also higher among elderly smokers 3,4 . Some data suggest that, as a group, the elderly not only smoke for a longer period of time than younger smokers, but are also less aware of the potential harms of tobacco use. As a result, they are more resistant to quit smoking, do not report a greater number of quitting attempts or methods, and tend not only to underestimate the risks but also to overestimate the benefits of smoking 3,5. Among the barriers that impact the success of smoking cessation attempts, the elderly have high rates of nicotine dependence 6 and are more likely to be dealing with life stresses and social isolation ${ }^{3}$. 
The need to gather information about tobacco consumption in vulnerable groups of individuals was previously reinforced as an important step for the development of appropriate intervention strategies to cessation 7 . Factors affecting tobacco use and its prevalence among the elderly are not completely understood. Understanding the values, beliefs, attitudes, and behaviors in relation to smoking among older individuals may help in the development of strategies that seek to reduce the associated morbidity and mortality. Consequently, the aim of this paper is to evaluate the prevalence of tobacco use among the elderly and to identify factors that are associated with variation in prevalence across studies.

\section{Method}

\section{Search strategy}

In order to gather as many studies as possible on the issue, the strategy for data collection aimed to identify papers which presented broad definitions of tobacco use, either everyday or some day use, regardless of the amount of smoking. The following sources were searched looking for cross-sectional studies: (i) electronic databases (MEDLINE, LILACS, and Biological Abstracts); (ii) hand-searching of specialist journals (journals most likely to contain surveys in this area have been searched such as Am J Public Health, Addiction, Tob Control, MMWR Morb Mortal Wkly Rep, and Prev Med); and (iii) cited reference searches.

As a first step, the search strategy used the following terms: (tobacco OR smok* OR cigarette) AND (elder* OR aged OR old) AND (epidemiology OR survey OR prevalence), which identified only three studies on the frequency of tobacco use in the elderly. In an attempt to find more studies, we performed a second search with the terms (tobacco OR smok* OR cigarette) AND (epidemiology OR survey OR prevalence).

\section{Inclusion/exclusion criteria}

All relevant surveys published between 1992 and 2004, with no language restrictions, were considered for this review. Surveys were eligible if they were conducted in the community with locally representative randomly selected samples, including persons from all age groups. Only data on the elderly were considered for the present review. Furthermore, studies were included if the questionnaires were designed to measure smoking habits with the definition specified in the text, no matter the type of use, amount and kind of tobacco product.
Studies were excluded for the following reasons: absence of aged subjects in the sample; lack of relevant information such as number of elderly people in the sample, prevalence rates of tobacco use in the elderly not reported; articles on tobacco-related medical conditions; review articles and technical impairment to access full text article (such as non availability on web, medical libraries, and at least two email contacts with the author).

\section{Data management}

The methodological procedures of the selected trials was assessed by two independent reviewers (V.M. and S.L.B.), who also extracted the data. In cases where the studies failed to describe the total elderly sample, or the method in use, the authors of the original studies were contacted for additional information (at least two email contacts with the author).

\section{Data extraction}

The country, year of data collection, socio-demographic measures, sample size of the elderly, prevalence rates, and methodological characteristics (such as type of interview and smoking definition) of the studies were extracted from the selected papers.

\section{Statistical analysis}

Studies which reported data from samples aged 60 years or more were analyzed together with those which reported data from samples with individuals above 65 years of age. We included studies which allowed the calculation of the standard error $(n=32)$. Homogeneity was evaluated by chi-squared test with significance level of 0.05 and I-squared statistic ${ }^{8}$. As the data were very heterogeneous, the random effects model was used. Analyses were carried out for both genders, together and separately, including other sociodemographic variables. Statistical analysis was performed using the "meta" command from Stata 10 program (Stata Corp., College Station, USA). Meta-regression was used to investigate variables associated to prevalence estimates.

\section{Results}

Of the 1,400 papers found, 160 abstracts were reviewed and 48 studies were included. We identified three epidemiological studies dealing with tobacco use among community-dwelling elderly $9,10,11$. The other 45 studies were carried out in 
community-living adults which included elderly samples. All these data were further checked reading the full paper.

Table 1 outlines the methodological characteristics of the included surveys.

Data were available from surveys carried out in all the continents and both north and south hemispheres. The adult population was largely assessed in all surveys, and the elderly subjects represented a small proportion of them. There was a wide variation in the number of participants aged 60 years and older included in the surveys, ranging from 5212 to 40,146 subjects 13 , and the total number of elderly subjects included in all surveys was 140,058 (median $=1,233$ ).

Questionnaire application was not homogeneous. Three studies were via telephone calls 12,14,15; three studies performed interviews both via telephone calls and personally in the household 16,17,18. Respondents were invited to attend a health center where they were interviewed in one survey 19, and in another one the questionnaire was either completed in a health center or sent to the respondents' houses 20 . The other 37 studies were solely conducted in household.

Information on adult current cigarette use was provided by the interviewee, except in nine surveys that allowed for proxy responses $11,13,18,21,22,23,24,25,26$.

Tobacco users were classified as smokers, regular smokers, tobacco smokers, cigarette smokers, current smokers, and hardcore and heavy smokers.

Definition of smokers were: (i) "those smoking every day" 27; (ii) "subjects who smoke daily or sometimes at the time of examination" 28,29,30,31; and (iii) "smoking one or more cigarettes weekly for 6 months or more before the survey" 32.

Definition of regular smokers included those people who: (i) "reported that they currently smoke daily or occasionally" 33; (ii) "those adults who smoked at least on a weekly basis" 34; (iii) Cigarette Smokers Regular light smokers - "those who smoked less than one packet a day" 22.

Current smokers were defined as those: (i) "smoking at least one cigarette each day" 19,35; (ii) "smoking one or more cigarettes daily for 6 months or more before the survey" 36,37; (iii) "smoking tobacco products at the time of the survey" 15,38,39,40,41,42; (iv) “having smoked at least 100 cigarettes in one's lifetime and currently smoke every day or some days or continue to smoke regularly or were still smoking daily at the time of the interview" 10,11,12,14,17,26,43,44,45,46,47; (v) individuals who gave positive response to the questions "Have you ever smoked regularly?", and "Do you currently smoke?”, or “Do you still smoke?” 48; (vi) "those who had smoked in the past month" 21; (vii) "respondents who reported that they had smoked in the three months prior to the survey" 49. Furthermore, some surveys considered current smokers as: (i) regular or daily smokers - "who at the time of the interview smoked at least one cigarette per day"; (ii) occasional smokers - "who at the time of interview did not smoke every day" 49,50; (iii) ever smokers - "more than 100 cigarettes in their lifetime" 51.

Heavy smokers were defined as: (i) "smokers with a daily consumption of more than $14 \mathrm{~g}$ of tobacco" 28; (ii) "individuals who consumed more than 25 cigarettes per day" 51; (iii) "those currently smoking $\geq 15$ cigarettes per day" 25 . Definition of hardcore smokers comprised people who answered: (i) "less than a day without cigarettes in the past five years" 7; (ii) "no attempt to give up smoking in the past 12 months" 7; (iii) no to "Do you want to give up smoking altogether?" 7; (iv) "no intention to give up smoking" 7; and (v) Regular heavy smokers - "those who smoked one packet or more daily" 22.

Some other definitions that were found included: (i) cigarette smoking - self-reported daily use of these products 52; (ii) tobacco smokers - "people who answered 'yes' to the question of smoking tobacco" 13; (iii) occasional smokers - "those who smoked less than one cigarette a day" 22. In some studies there was no definition of smoking status and smokers were classified as current smokers 20,23,53,54. Data derived from these less specific definitions were analyzed as "No definition", therefore separately from the better delineated definitions listed above.

In three surveys, definition of tobacco use included local tobacco products such as beedies 44 , toombak 55 , waterpipe 22 , and one included other forms of tobacco use such as chewing of tobacco 13 . Data on local and non-inhaled tobacco products were not included for analysis.

Overall prevalence of tobacco use was higher for men. Smoking prevalence among males was highest in Indonesia (84.5\%) 21 , and lowest in Australia (11\%) 34. Among females, the smoking prevalence was highest in Tonga (26.1\%) 49, and lowest in Kazakhstan (0.4\%) 35 and Bulgaria (0\%) 27. Some studies described prevalence rates for both genders and rates varied from $6.8 \%$ in the United States 17 to $63 \%$ in Copenhagen, Denmark 28.

Meta-analysis results can be seen in Figures 1, 2 and 3. A statistical procedure confirmed the initial assumption of a greater prevalence in men, with an overall prevalence of $13.5 \%$ (95\%CI: 12.0 15.1). Smoking prevalence rates by gender were $22.5 \%(17.0-28.1)$ and $8.7 \%$ (6.8-10.6) for men and women, respectively. The chi-squared test for homogeneity and I-squared statistic showed 
Table 1

Methodological characteristics of included surveys.

\begin{tabular}{|c|c|c|c|c|c|c|c|}
\hline Author (year) & Methods & Setting & $\begin{array}{c}\text { Proxy } \\
\text { interviews }\end{array}$ & $\begin{array}{l}\text { Definition of } \\
\text { smoking status }\end{array}$ & Participants & $\begin{array}{c}\text { Elderly } \\
\text { participants }\end{array}$ & $\begin{array}{l}\text { Prevalence of } \\
\text { tobacco use }\end{array}$ \\
\hline $\begin{array}{l}\text { Gilmore } \\
\text { et al. } 35 \text { (2004) }\end{array}$ & $\begin{array}{c}\text { Questionnaire } \\
\text { survey }\end{array}$ & $\begin{array}{l}\text { USSR: household } \\
\text { interview }\end{array}$ & No & $\begin{array}{l}\text { Current smokers: } \\
\text { respondents } \\
\text { reporting } \\
\text { currently smoking } \\
\text { at least } 1 \\
\text { cigarette per day }\end{array}$ & $\begin{array}{c}18,428 \\
\text { participants } \\
\text { aged } \geq \\
18 \text { years }\end{array}$ & $\begin{array}{c}\text { Participants } \\
\text { aged } \geq \\
60 \text { years: } \\
\text { Armenia: 560; } \\
\text { Belarus: 558; } \\
\text { Georgia: 558; } \\
\text { Kazakhstan: } \\
\text { 376; } \\
\text { Kyrgyzstan: } \\
\text { 318; Moldova: } \\
\text { 520; Russia: } \\
\text { 1,118; Ukraine: } \\
852\end{array}$ & $\begin{array}{c}\geq 60 \text { years. Total } \\
\text { sample (M: 37\%/W: } \\
\text { 1.5\%), Armenia (M: } \\
\text { 44.4\%/W: 1\%), Belarus } \\
\text { (M: 40.3\%/W: 0.9\%), } \\
\text { Georgia (M: 33.9\%/W: } \\
\text { 3.4\%), Kazakhstan } \\
\text { (M: 50\%/W: 0.4\%), } \\
\text { Kyrgyzstan (M: 25\%/W: } \\
\text { 1.7\%), Moldova (M: } \\
\text { 24.7\%/W: 1.1\%), Russia } \\
\text { (M: 42.3\%/W: } 2.5 \%), \\
\text { Ukraine (M: } 35.7 \% / \mathrm{W}: \\
1 \%)\end{array}$ \\
\hline INCA 47 (2004) & $\begin{array}{c}\text { Questionnaire } \\
\text { survey }\end{array}$ & $\begin{array}{c}\text { Brazil (Manaus, } \\
\text { Belém, } \\
\text { Fortaleza, Natal, } \\
\text { João Pessoa, } \\
\text { Recife, Aracaju, } \\
\text { Campo Grande, } \\
\text { Distrito Federal, } \\
\text { Belo Horizonte, } \\
\text { Vitória, Rio de } \\
\text { Janeiro, São } \\
\text { Paulo, Curitiba, } \\
\text { Florianópolis, } \\
\text { Porto Alegre): } \\
\text { household } \\
\text { survey }\end{array}$ & No & $\begin{array}{l}\text { Current smokers: } \\
\text { persons who } \\
\text { reported both } \\
\text { having smoked } \\
\geq 100 \text { cigarettes } \\
\text { during their } \\
\text { lifetime and } \\
\text { currently smokes } \\
\text { every day or } \\
\text { some days }\end{array}$ & $\begin{array}{l}23,457 \\
\text { participants aged } \\
\geq 15 \text { years }\end{array}$ & $\begin{array}{c}\text { Participants } \\
\text { aged } \geq 60 \\
\text { years: Manaus: } \\
\text { 142; Belém: } \\
\text { 145; Fortaleza: } \\
\text { 270; Natal: 95; } \\
\text { João Pessoa: } \\
\text { 140; Recife: } \\
\text { 147; Aracaju: } \\
\text { 83; Campo } \\
\text { Grande: } \\
\text { 69; Distrito } \\
\text { Federal: } \\
\text { 154; Belo } \\
\text { Horizonte: } 269 ; \\
\text { Vitória: 83; Rio } \\
\text { de Janeiro: } \\
\text { 460; São } \\
\text { Paulo: 118; } \\
\text { Curitiba: } 242 ; \\
\text { Florianópolis: } \\
\text { 112; Porto } \\
\text { Alegre: 236 }\end{array}$ & $\begin{array}{c}\text { z60 years: Manaus } \\
\text { (M: 21.7\%/W: 11\%), } \\
\text { Belém (M: 18.6\%/W: } \\
\text { 3.5\%), Fortaleza (M: } \\
\text { 21.1\%/W: 8.7\%), Natal } \\
\text { (M: 21.1\%/W: 3.5\%), } \\
\text { João Pessoa (M: } \\
\text { 15.4\%/W: 6.8\%), Recife } \\
\text { (M: 21.2\%/W: 7.4\%), } \\
\text { Aracaju (M: 21.2\%/W: } \\
\text { 8\%), Campo Grande } \\
\text { (M: 15.2\%/W: 11.1\%), } \\
\text { Distrito Federal (M: } \\
\text { 15.9\%/W: 9.9\%), Belo } \\
\text { Horizonte (M: 15.2\%/W: } \\
\text { 2.8\%), Vitória (M: } \\
\text { 8.6\%/W: 8.3\%), Rio de } \\
\text { Janeiro (M: 16.2\%/W: } \\
\text { 8.2\%), São Paulo } \\
\text { (M: 24.1\%/W: 9.4\%), } \\
\text { Curitiba (M: 20.8\%/W: } \\
\text { 14.7\%), Florianópolis } \\
\text { (M: 12.5\%/W: 8.3\%), } \\
\text { Porto Alegre (M: } \\
\text { 19.6\%/W: 12.5\%) }\end{array}$ \\
\hline $\begin{array}{l}\text { Lima-Costa } 24 \\
(2004)\end{array}$ & $\begin{array}{c}\text { Questionnaire } \\
\text { survey }\end{array}$ & $\begin{array}{l}\text { Brazil: household } \\
\text { interview }\end{array}$ & $\begin{array}{l}\text { Yes - proxy } \\
\text { interviews } \\
\text { represented } \\
28 \% \text { of total } \\
\text { sample }\end{array}$ & Current smokers & $\begin{array}{l}13,701 \text { both sex } \\
\text { aged } \geq 20 \text { years }\end{array}$ & $\begin{array}{c}1,774 \\
\text { participants } \\
\text { aged } \geq 60 \\
\text { years }\end{array}$ & $\begin{array}{c}\geq 60 \text { years }(12.8 \% \text { both } \\
\text { sex })\end{array}$ \\
\hline
\end{tabular}

(continues) 
Table 1 (continued)

\begin{tabular}{|c|c|c|c|c|c|c|c|}
\hline Author (year) & Methods & Setting & $\begin{array}{c}\text { Proxy } \\
\text { interviews }\end{array}$ & $\begin{array}{c}\text { Definition of } \\
\text { smoking status }\end{array}$ & Participants & $\begin{array}{c}\text { Elderly } \\
\text { participants }\end{array}$ & $\begin{array}{l}\text { Prevalence of } \\
\text { tobacco use }\end{array}$ \\
\hline $\begin{array}{l}\text { Santos \& } \\
\text { Barros } 51 \\
(2004)\end{array}$ & $\begin{array}{c}\text { Questionnaire } \\
\text { survey }\end{array}$ & $\begin{array}{l}\text { Portugal: } \\
\text { household } \\
\text { interview }\end{array}$ & No & $\begin{array}{l}\text { Current smokers: } \\
\text { included both } \\
\text { daily (at least } \\
1 \text { cigarette per } \\
\text { day at the time } \\
\text { of the survey) } \\
\text { and occasional } \\
\text { smokers (less } \\
\text { than a cigarette } \\
\text { per day) }\end{array}$ & $\begin{array}{c}1,690 \text { participants } \\
\text { both sex aged } \geq \\
18 \text { years }\end{array}$ & - & $\begin{array}{c}60-69 \text { years }(\mathrm{M}: \\
26.2 \% / \mathrm{W}: 4.5 \%), \geq 70 \\
\text { years (M: } 13.9 \% / \mathrm{W}: \\
1.4 \%)\end{array}$ \\
\hline $\begin{array}{l}\text { Villabí et al. } 31 \\
\text { (2004) }\end{array}$ & $\begin{array}{c}\text { Questionnaire } \\
\text { survey }\end{array}$ & $\begin{array}{l}\text { Spain: household } \\
\text { interview }\end{array}$ & No & $\begin{array}{l}\text { Smokers: } \\
\text { included both } \\
\text { daily and } \\
\text { occasional } \\
\text { smokers }\end{array}$ & $\begin{array}{c}8,833 \text { participants } \\
\text { both sex aged } \geq \\
15 \text { years }\end{array}$ & $\begin{array}{c}2,140 \\
\text { participants } \\
\text { aged } \geq 64 \\
\text { years (M: } 827 \\
\text { men/W: } 1,314 \text { ) }\end{array}$ & $\begin{array}{c}\geq 64 \text { years }(\mathrm{M}: \\
20.4 \% / \mathrm{W}: 2 \% / \text { both sex: } \\
9.2 \%)\end{array}$ \\
\hline $\begin{array}{l}\text { Jarvis et al. } 6 \\
\text { (2003) }\end{array}$ & $\begin{array}{c}\text { Questionnaire } \\
\text { survey }\end{array}$ & $\begin{array}{l}\text { England: } \\
\text { household } \\
\text { interview }\end{array}$ & No & $\begin{array}{l}\text { Hardcore smoker: } \\
\text { less than a } \\
\text { day without } \\
\text { cigarettes in the } \\
\text { past } 5 \text { years; } \\
\text { no attempt to } \\
\text { give up smoking } \\
\text { in the past } 12 \\
\text { months; no to } \\
\text { "Do you want to } \\
\text { give up smoking } \\
\text { altogether?"; no } \\
\text { intention to give } \\
\text { up smoking }\end{array}$ & $\begin{array}{l}\text { 7,766 both sex } \\
\text { cigarette smokers } \\
\text { aged } \geq 16 \text { years }\end{array}$ & $\begin{array}{c}1,083 \\
\text { participants } \\
\text { aged } \geq 65 \\
\text { years }\end{array}$ & $\begin{array}{c}\geq 65 \text { years ( } 16 \% \text { both } \\
\text { sex/5\% both sex } \\
\text { hardcore smoking) }\end{array}$ \\
\hline $\begin{array}{l}\text { Lau et al. } 15 \\
\text { (2003) }\end{array}$ & $\begin{array}{c}\text { Questionnaire } \\
\text { survey }\end{array}$ & $\begin{array}{c}\text { Hong Kong, } \\
\text { China: telephone } \\
\text { interview }\end{array}$ & No & $\begin{array}{l}\text { Current smokers: } \\
\text { smoking tobacco } \\
\text { products at } \\
\text { the time of the } \\
\text { survey }\end{array}$ & $\begin{array}{l}39,963 \text { women } \\
\text { aged } \geq 18 \text { years }\end{array}$ & $\begin{array}{l}\text { 6,176 women } \\
\text { aged } \geq 61 \\
\text { years }\end{array}$ & $\begin{array}{c}61-65 \text { years }(1.9 \%), 66- \\
70 \text { years }(2.4 \%),>70 \\
\text { years }(2.7 \%)\end{array}$ \\
\hline $\begin{array}{l}\text { Rani et al. }{ }^{13} \\
(2003)\end{array}$ & $\begin{array}{c}\text { Questionnaire } \\
\text { survey }\end{array}$ & $\begin{array}{c}\text { India: household } \\
\text { interview }\end{array}$ & $\begin{array}{l}\text { Yes - the } \\
\text { questionnaire } \\
\text { was } \\
\text { administered } \\
\text { to the } \\
\text { head of the } \\
\text { household or } \\
\text { to any other } \\
\text { competent } \\
\text { adult member } \\
\text { of the } \\
\text { household }\end{array}$ & $\begin{array}{l}\text { Chewing of } \\
\text { tobacco: those } \\
\text { people who } \\
\text { answered "yes" } \\
\text { to the question } \\
\text { on chewing } \\
\text { tobacco/ } \\
\text { Tobacco } \\
\text { smokers: those } \\
\text { people who } \\
\text { answered "yes" } \\
\text { to the question of } \\
\text { smoking tobacco }\end{array}$ & $\begin{array}{c}334,553 \\
\text { participants both } \\
\text { sex aged } \geq 15 \\
\text { years }\end{array}$ & $\begin{array}{c}40,146 \\
\text { participants } \\
\text { aged } \geq 60 \\
\text { years }\end{array}$ & $\begin{array}{c}\text { Tobacco smoking: } \geq \\
60 \text { years (M: } 38.1 \% / \mathrm{W}: \\
5.2 \%) \text {; chewing of } \\
\text { tobacco: } \geq 60 \text { years (M: } \\
22.4 \% / \mathrm{W}: 37.4 \%)\end{array}$ \\
\hline
\end{tabular}

(continues) 
Table 1 (continued)

\begin{tabular}{|c|c|c|c|c|c|c|c|}
\hline Author (year) & Methods & Setting & $\begin{array}{c}\text { Proxy } \\
\text { interviews }\end{array}$ & $\begin{array}{c}\text { Definition of } \\
\text { smoking status }\end{array}$ & Participants & $\begin{array}{c}\text { Elderly } \\
\text { participants }\end{array}$ & $\begin{array}{l}\text { Prevalence of } \\
\text { tobacco use }\end{array}$ \\
\hline $\begin{array}{l}\text { Shapo et al. } 19 \\
(2003)\end{array}$ & $\begin{array}{c}\text { Questionnaire } \\
\text { survey }\end{array}$ & $\begin{array}{l}\text { Albania: } \\
\text { respondents } \\
\text { were invited to } \\
\text { attend a health } \\
\text { center in Tirana } \\
\text { were they were } \\
\text { interviewed }\end{array}$ & No & $\begin{array}{l}\text { Current smokers: } \\
\text { smoking at least } \\
\text { one cigarette } \\
\text { each day }\end{array}$ & $\begin{array}{c}1,120 \text { participants } \\
\text { both sex aged } \geq \\
25 \text { years }\end{array}$ & $\begin{array}{c}229 \\
\text { participants } \\
\text { aged } \geq 65 \\
\text { years; } 135 \\
(25.2 \%) \text { men } \\
\text { and } 94(16.1 \%) \\
\text { women }\end{array}$ & $\begin{array}{c}\geq 65 \text { years }(\mathrm{M}: \\
26.7 \% / \mathrm{W}: 18.1 \% / \text { both } \\
\text { sex: } 23.1 \%)\end{array}$ \\
\hline $\begin{array}{l}\text { White et al. } 34 \\
\text { (2003) }\end{array}$ & $\begin{array}{c}\text { Questionnaire } \\
\text { survey }\end{array}$ & $\begin{array}{l}\text { Australia: } \\
\text { household } \\
\text { interview }\end{array}$ & No & $\begin{array}{l}\text { Regular smokers: } \\
\text { those adults who } \\
\text { smoked at least } \\
\text { on a weekly basis }\end{array}$ & $\begin{array}{c}\text { All samples } \\
\text { included both sex } \\
\text { participants aged } \\
\geq 18 \text { years. } 1980 \\
\text { sample: } 3,696 ; \\
1983 \text { sample: } \\
\text { 5,087; } 1986 \\
\text { sample: } 8,513 ; \\
\text { 1989 sample: } \\
\text { 5,314; } 1992 \\
\text { sample: } 6,304 ; \\
\text { 1995 sample: } \\
\text { 5,149; } 1998 \\
\text { sample: } 7,852 ; \\
2001 \text { sample: } \\
23,376\end{array}$ & $\begin{array}{c}\text { Aged } \geq 60 \\
\text { years. } 1980 \\
\text { sample: } 733 ; \\
1983 \text { sample: } \\
\text { 1,002; } 1986 \\
\text { sample: } 1,819 ; \\
1989 \text { sample: } \\
\text { 1,175; } 1992 \\
\text { sample: } 1,386 ; \\
\text { 1995 sample: } \\
\text { 1,414; } 1998 \\
\text { sample: } 1,928 ; \\
2001 \text { sample: } \\
4,992\end{array}$ & $\begin{array}{l}\text { z60 years. } 1980 \\
\text { sample: (M: } 22 \% / \mathrm{W}: \\
\text { 18\%/both sex: } 20 \%), \\
\text { 1983 sample (M: } \\
\text { 26\%/W: } 17 \% / \text { both sex: } \\
\text { 21\%), } 1986 \text { sample (M: } \\
\text { 22\%/W: } 17 \% / \text { both sex: } \\
\text { 19\%), } 1989 \text { sample (M: } \\
\text { 18\%/W: } 15 \% / \text { both sex: } \\
\text { 17\%), } 1992 \text { sample (M: } \\
\text { 16\%/W: } 12 \% / \text { both sex: } \\
\text { 14\%), } 1995 \text { sample (M: } \\
\text { 15\%/W: } 12 \% / \text { both sex: } \\
\text { 13\%), } 1998 \text { sample (M: } \\
\text { 12\%/W: } 12 \% / \text { both sex: } \\
\text { 12\%), } 2001 \text { sample (M: } \\
\text { 11\%/W: } 8 \% / \text { both sex: } \\
10 \% \text { ) }\end{array}$ \\
\hline $\begin{array}{l}\text { Woollery et al. } 46 \\
\text { (2003) }\end{array}$ & $\begin{array}{c}\text { Questionnaire } \\
\text { survey }\end{array}$ & $\begin{array}{l}\text { USA: household } \\
\text { interview. Data } \\
\text { from the NHIS }\end{array}$ & No & $\begin{array}{l}\text { Current smokers: } \\
\text { persons who } \\
\text { reported both } \\
\text { having smoked } \\
\geq 100 \text { cigarettes } \\
\text { during their } \\
\text { lifetime and } \\
\text { currently smokes } \\
\text { every day or } \\
\text { some days }\end{array}$ & $\begin{array}{c}\text { NHIS 2001: } \\
33,326 \\
\text { participants both } \\
\text { sex aged } \geq 18 \\
\text { years }\end{array}$ & & $\begin{array}{l}\geq 65 \text { years }(10.1 \% \text { both } \\
\text { sex })\end{array}$ \\
\hline $\begin{array}{l}\text { Bursac et al. } 14 \\
\text { (2002) }\end{array}$ & $\begin{array}{c}\text { Questionnaire } \\
\text { survey }\end{array}$ & $\begin{array}{c}\text { Oklahoma } \\
\text { (USA): telephone } \\
\text { interview. Data } \\
\text { from the BRFSS, } \\
\text { Oklahoma } \\
\text { BRFSS, } \\
\text { Oklahoma } \\
\text { REACH } 2010 \\
\text { BRFS }\end{array}$ & $\begin{array}{c}\text { No } \\
\text { information } \\
\text { about proxy } \\
\text { interviews }\end{array}$ & $\begin{array}{l}\text { Cigarette smoker: } \\
\text { individual that } \\
\text { smoked } 100 \text { or } \\
\text { more cigarettes } \\
\text { in their lifetime } \\
\text { and currently } \\
\text { smokes every day } \\
\text { or some day }\end{array}$ & $\begin{array}{l}3,732 \text { surveys } \\
\text { from } 7 \text { strata }\end{array}$ & & $\begin{array}{c}\geq 65 \text { years ( } 16.4 \% \text { both } \\
\text { sex American Indians/ } \\
\text { Alaska Natives) }\end{array}$ \\
\hline $\begin{array}{l}\text { Steyn et al. } 33 \\
\text { (2002) }\end{array}$ & $\begin{array}{c}\text { Questionnaire } \\
\text { survey }\end{array}$ & $\begin{array}{c}\text { South Africa: } \\
\text { household } \\
\text { interview }\end{array}$ & No & $\begin{array}{l}\text { Regular } \\
\text { smokers: those } \\
\text { people who } \\
\text { reported that } \\
\text { they currently } \\
\text { smoke daily or } \\
\text { occasionally }\end{array}$ & $\begin{array}{c}13,826 \\
\text { participants both } \\
\text { sex aged } \geq 15 \\
\text { years }\end{array}$ & $\begin{array}{c}1,473 \text { aged } \geq \\
65 \text { years }\end{array}$ & $\begin{array}{c}\geq 65 \text { years }(\mathrm{M}: \\
38.5 \% / \mathrm{W}: 7.6 \%)\end{array}$ \\
\hline
\end{tabular}

(continues) 
Table 1 (continued)

\begin{tabular}{|c|c|c|c|c|c|c|c|}
\hline Author (year) & Methods & Setting & $\begin{array}{c}\text { Proxy } \\
\text { interviews }\end{array}$ & $\begin{array}{l}\text { Definition of } \\
\text { smoking status }\end{array}$ & Participants & $\begin{array}{c}\text { Elderly } \\
\text { participants }\end{array}$ & $\begin{array}{l}\text { Prevalence of } \\
\text { tobacco use }\end{array}$ \\
\hline $\begin{array}{l}\text { Gilmore et al. } 48 \\
\text { (2001) }\end{array}$ & $\begin{array}{c}\text { Questionnaire } \\
\text { survey }\end{array}$ & $\begin{array}{l}\text { Ukraine: } \\
\text { household } \\
\text { interview }\end{array}$ & No & $\begin{array}{c}\text { Current and } \\
\text { previous } \\
\text { smoking: subjects } \\
\text { who gave a } \\
\text { positive reply to } \\
\text { the questions: } \\
\text { "Have you ever } \\
\text { smoked?" and } \\
\text { "Do you still } \\
\text { smoke?" }\end{array}$ & $\begin{array}{c}1,590 \text { participants } \\
\text { both sex aged } \geq \\
18 \text { years }\end{array}$ & & $\begin{array}{c}\geq 60 \text { years (M: } \\
32.6 \% / \mathrm{W}: 0.8 \%)\end{array}$ \\
\hline $\begin{array}{l}\text { Gilmore et al. } 53 \\
\text { (2001) }\end{array}$ & $\begin{array}{c}\text { Questionnaire } \\
\text { survey }\end{array}$ & $\begin{array}{l}\text { Belarus: } \\
\text { household } \\
\text { interview }\end{array}$ & No & Current smokers & $\begin{array}{c}1,090 \text { participants } \\
\text { both sex aged } \geq \\
18 \text { years }\end{array}$ & $\begin{array}{c}269 \text { aged } \geq 60 \\
\text { years }\end{array}$ & $\begin{array}{l}\geq 60 \text { years }(\mathrm{M}: \\
41.9 \% / \mathrm{W}: 0.7 \%)\end{array}$ \\
\hline $\begin{array}{l}\text { Lima-Costa } \\
\text { et al. }{ }^{11}(2001)\end{array}$ & $\begin{array}{c}\text { Questionnaire } \\
\text { survey }\end{array}$ & $\begin{array}{c}\text { Bambuí (Brazil): } \\
\text { household } \\
\text { interview }\end{array}$ & $\begin{array}{l}\text { Yes - proxy } \\
\text { interviews } \\
\text { represented } \\
5.6 \% \text { of total } \\
\text { sample }\end{array}$ & $\begin{array}{l}\text { Current smokers: } \\
\text { subjects who } \\
\text { reported having } \\
\text { smoked } \geq 100 \\
\text { cigarettes during } \\
\text { their lifetime and } \\
\text { who currently } \\
\text { smoked }\end{array}$ & $\begin{array}{c}1,606 \text { participants } \\
\text { both sex aged } \geq \\
60 \text { years }\end{array}$ & $\begin{array}{c}1,606 \text { aged } \geq \\
60 \text { years }\end{array}$ & $\begin{array}{c}\geq 60 \text { years }(18.7 \% \text { both } \\
\text { sex })\end{array}$ \\
\hline $\begin{array}{l}\text { Nasir \& Rehan } 44 \\
\text { (2001) }\end{array}$ & $\begin{array}{c}\text { Questionnaire } \\
\text { survey }\end{array}$ & $\begin{array}{l}\text { Pakistan: } \\
\text { household } \\
\text { interview }\end{array}$ & No & $\begin{array}{l}\text { Smoker: smoked } \\
\text { more than } 100 \\
\text { cigarettes or } \\
\text { beedies in the } \\
\text { lifetime and was } \\
\text { still smoking }\end{array}$ & $\begin{array}{c}13,104 \\
\text { participants both } \\
\text { sex aged } \geq 8 \\
\text { years }\end{array}$ & $\begin{array}{c}818 \text { aged } \geq 65 \\
\text { years }\end{array}$ & $\begin{array}{l}\geq 65 \text { years. Regular } \\
\text { smokers (M: } 38.5 \% / \mathrm{W}: \\
7.6 \% \text { ), daily smokers } \\
\text { (M: } 35.4 \% / \mathrm{W}: 6.6 \% \text { ) }\end{array}$ \\
\hline $\begin{array}{l}\text { Ohida et al. } 42 \\
\text { (2001) }\end{array}$ & $\begin{array}{c}\text { Questionnaire } \\
\text { survey }\end{array}$ & $\begin{array}{l}\text { Japan: self- } \\
\text { administered } \\
\text { questionnaire }\end{array}$ & No & $\begin{array}{l}\text { Current smokers: } \\
\text { smoking tobacco } \\
\text { products at } \\
\text { the time of the } \\
\text { survey }\end{array}$ & $\begin{array}{c}38,710 \\
\text { participants both } \\
\text { sex aged } \geq 15 \\
\text { years }\end{array}$ & $\begin{array}{c}8,432 \text { aged } \\
\geq 60 \text { years } \\
\text { (M: } 3,655 / \mathrm{W}: \\
4,777)\end{array}$ & $\begin{array}{c}\geq 60 \text { years (M: } \\
42.9 \% / \mathrm{W}: 7.7 \%)\end{array}$ \\
\hline $\begin{array}{l}\text { Shah et al. } 45 \\
\text { (2001) }\end{array}$ & $\begin{array}{c}\text { Questionnaire } \\
\text { survey }\end{array}$ & $\begin{array}{l}\text { Ghizar District } \\
\text { (Pakistan): } \\
\text { household } \\
\text { interview }\end{array}$ & No & $\begin{array}{c}\text { Cigarette } \\
\text { smokers: } \\
\text { respondents who } \\
\text { were currently } \\
\text { smoking and who } \\
\text { had smoked } \geq \\
100 \text { cigarettes in } \\
\text { their lifetime }\end{array}$ & $\begin{array}{c}4,203 \text { participants } \\
\text { both sex aged } \geq \\
18 \text { years }\end{array}$ & $\begin{array}{c}562 \text { aged } \geq 60 \\
\text { years }\end{array}$ & $\begin{array}{c}\geq 60 \text { years }(\mathrm{M}: \\
25.4 \% / \mathrm{W}: 18.3 \%)\end{array}$ \\
\hline $\begin{array}{l}\text { Clausen et al. } 9 \\
(2000)\end{array}$ & $\begin{array}{c}\text { Questionnaire } \\
\text { survey }\end{array}$ & $\begin{array}{l}\text { Mmankgodi } \\
\text { Village } \\
\text { (Botswana): } \\
\text { household } \\
\text { interview }\end{array}$ & No & $\begin{array}{l}\text { Daily smokers: } \\
\text { those reporting } \\
\text { smoking on a } \\
\text { daily basis }\end{array}$ & $\begin{array}{c}337 \text { participants } \\
\text { both sex aged } \geq \\
60 \text { years }\end{array}$ & $\begin{array}{c}337 \text { aged } \geq 60 \\
\text { years }\end{array}$ & $\begin{array}{c}\geq 60 \text { years }(11 \% \\
\text { both sex })\end{array}$ \\
\hline
\end{tabular}

(continues) 


\begin{tabular}{|c|c|c|c|c|c|c|c|}
\hline Author (year) & Methods & Setting & $\begin{array}{c}\text { Proxy } \\
\text { interviews }\end{array}$ & $\begin{array}{c}\text { Definition of } \\
\text { smoking status }\end{array}$ & Participants & $\begin{array}{c}\text { Elderly } \\
\text { participants }\end{array}$ & $\begin{array}{l}\text { Prevalence of } \\
\text { tobacco use }\end{array}$ \\
\hline $\begin{array}{l}\text { Cox et al. } 38 \\
(2000)\end{array}$ & $\begin{array}{l}\text { Questionnaire } \\
\text { survey }\end{array}$ & $\begin{array}{l}\text { Mauritius: } \\
\text { household } \\
\text { interview, } \\
3 \text { samples } \\
\text { collected }\end{array}$ & No & $\begin{array}{l}\text { Smokers: those } \\
\text { reporting current } \\
\text { smoking at the } \\
\text { time of survey }\end{array}$ & $\begin{array}{c}1987 \text { sample: } \\
5,072 \\
\text { participants, } \\
\text { both sex, aged } \\
25-74 \text { years; } 1992 \\
\text { sample: } 6,573 \\
\text { participants, } \\
\text { both sex, aged } \\
\geq 25 \text { years; } 1998 \\
\text { sample: } 6,281 \\
\text { participants, both } \\
\text { sex, aged } \geq 20 \\
\text { years }\end{array}$ & & $\begin{array}{c}1987 \text { sample: } 60-69 \\
\text { years (M: } 54 \% / \mathrm{W}: \\
7.7 \%), \geq 70 \text { years (M: } \\
\text { 49.3\%/W: } 11.9 \%) ; 1992 \\
\text { sample: } 60-69 \text { years } \\
\text { (M: } 35.8 \% / \mathrm{W}: 5.6 \%), \\
\geq 70 \text { years (M: } 36 \% / \mathrm{W}: \\
\text { 3.9\%); } 1998 \text { sample: } 60- \\
69 \text { years (M: } 36.2 \% / \mathrm{W}: \\
3.6 \%), \geq 70 \text { years }(\mathrm{M}: \\
26.8 \% / \mathrm{W}: 2.3 \%)\end{array}$ \\
\hline $\begin{array}{l}\text { Hu \& Tsai } 41 \\
(2000)\end{array}$ & $\begin{array}{c}\text { Questionnaire } \\
\text { survey }\end{array}$ & $\begin{array}{l}\text { China: } \\
\text { household } \\
\text { interview }\end{array}$ & Not informed & $\begin{array}{l}\text { Current smokers: } \\
\text { smoking } \\
\text { cigarettes at } \\
\text { the time of the } \\
\text { interview }\end{array}$ & $\begin{array}{c}24,996 \\
\text { participants both } \\
\text { sex aged } \geq 15 \\
\text { years }\end{array}$ & & $\begin{array}{c}\geq 60 \text { years }(32.4 \% \text { both } \\
\text { sex) }\end{array}$ \\
\hline $\begin{array}{l}\text { Jarallah et al. } 36 \\
\text { (1999) }\end{array}$ & $\begin{array}{l}\text { Questionnaire } \\
\text { survey }\end{array}$ & $\begin{array}{c}\text { Saudi Arabia: } \\
\text { household } \\
\text { interview }\end{array}$ & No & $\begin{array}{l}\text { Current smokers: } \\
\text { smoking one or } \\
\text { more cigarettes } \\
\text { daily for } 6 \\
\text { months or more } \\
\text { before the survey }\end{array}$ & $\begin{array}{c}8,310 \text { participants } \\
\text { both sex aged } \geq \\
15 \text { years }\end{array}$ & $\begin{array}{l}1,230 \text { aged } \geq \\
61 \text { years }\end{array}$ & $\begin{array}{c}\geq 61 \text { years }(8 \% \text { both } \\
\text { sex) }\end{array}$ \\
\hline $\begin{array}{l}\text { Kamimoto } \\
\text { et al. } 17 \text { (1999) }\end{array}$ & $\begin{array}{c}\text { Questionnaire } \\
\text { survey }\end{array}$ & $\begin{array}{l}\text { USA: data from } \\
\text { the BRFSS and } \\
\text { from the NHIS }\end{array}$ & No & $\begin{array}{l}\text { Current smokers: } \\
\text { persons who } \\
\text { reported smoking } \\
\geq 100 \text { cigarettes } \\
\text { during their } \\
\text { lifetime and who } \\
\text { currently smoke } \\
\text { every day or } \\
\text { some days }\end{array}$ & $\begin{array}{l}\text { NHIS 1993- } \\
\text { 1995: } 17,754 \\
\text { participants, } \\
\text { both sex, aged } \\
\geq 55 \text { years; } \\
\text { BRFSS 1995- } \\
\text { 1997: } 116,690 \\
\text { participants, both } \\
\text { sex, aged } \geq 55 \\
\text { years }\end{array}$ & & $\begin{array}{l}\text { NHIS sample: } 65-74 \\
\text { years ( } 15.2 \% \text { both sex), } \\
\geq 75 \text { years ( } 8.4 \% \text { both } \\
\text { sex); BRFSS sample: } 65- \\
74 \text { years ( } 13.3 \% \text { both } \\
\text { sex), } \geq 75 \text { years }(6.8 \% \\
\text { both sex) }\end{array}$ \\
\hline $\begin{array}{l}\text { Markides et al. } 10 \\
\text { (1999) }\end{array}$ & $\begin{array}{c}\text { Questionnaire } \\
\text { survey }\end{array}$ & $\begin{array}{l}\text { USA: household } \\
\text { interview. } \\
\text { Data from the } \\
\text { H-EPESE and the } \\
\text { HHANES }\end{array}$ & No & $\begin{array}{l}\text { Current smokers: } \\
\text { ever smokers } \\
\text { (those who } \\
\text { smoked at least } \\
100 \text { cigarettes } \\
\text { in their lifetime) } \\
\text { who were current } \\
\text { smokers at the } \\
\text { time of the } \\
\text { survey }\end{array}$ & $\begin{array}{l}\text { HHANES } 1982- \\
\text { 1984: } 753 \\
\text { participants, both } \\
\text { sex, aged } \geq 55 \\
\text { years; H-EPESE } \\
\text { 1993-1994: 2,890 } \\
\text { participants, both } \\
\text { sex, aged } \geq 65 \\
\text { years }\end{array}$ & $\begin{array}{l}\text { HHANES 1982- } \\
\text { 1984: } 247 \\
\text { participants, } \\
\text { both sex, aged } \\
\geq 65 \text { years; } \\
\text { H-EPESE 1993- } \\
\text { 1994: } 2,890 \\
\text { participants, } \\
\text { both sex, aged } \\
\geq 65 \text { years }\end{array}$ & $\begin{array}{c}\text { HHANES sample: 65- } \\
74 \text { years (M: 41.2\%/W: } \\
\text { 19.2\%); H-EPESE 1993- } \\
\text { 1994: 65-74 years (M: } \\
\text { 19.6\%/W: 9.8\%), 75-84 } \\
\text { years (M: } 15.8 \% / \mathrm{W}: \\
6.6 \%)\end{array}$ \\
\hline
\end{tabular}

(continues) 
Table 1 (continued)

\begin{tabular}{|c|c|c|c|c|c|c|c|}
\hline Author (year) & Methods & Setting & $\begin{array}{c}\text { Proxy } \\
\text { interviews }\end{array}$ & $\begin{array}{c}\text { Definition of } \\
\text { smoking status }\end{array}$ & Participants & $\begin{array}{c}\text { Elderly } \\
\text { participants }\end{array}$ & $\begin{array}{l}\text { Prevalence of } \\
\text { tobacco use }\end{array}$ \\
\hline $\begin{array}{l}\text { Yang et al. } 40 \\
\text { (1999) }\end{array}$ & $\begin{array}{c}\text { Questionnaire } \\
\text { survey }\end{array}$ & $\begin{array}{l}\text { China: } \\
\text { household } \\
\text { interview }\end{array}$ & No & $\begin{array}{l}\text { Current smokers: } \\
\text { those smoking } \\
\text { tobacco products } \\
\text { at the time of the } \\
\text { survey }\end{array}$ & $\begin{array}{c}120,298 \\
\text { participants, both } \\
\text { sex, aged 15-69 } \\
\text { years }\end{array}$ & $\begin{array}{l}13,628 \text { aged } \\
60-69 \text { years }\end{array}$ & $\begin{array}{c}\text { 60-69 years } \\
\text { (35\% both sexo }\end{array}$ \\
\hline $\begin{array}{l}\text { Arnett et al. } 52 \\
\text { (1998) }\end{array}$ & $\begin{array}{c}\text { Questionnaire } \\
\text { survey }\end{array}$ & $\begin{array}{c}\text { Minnesota (USA): } \\
\text { household } \\
\text { interview }\end{array}$ & No & $\begin{array}{l}\text { Current smokers: } \\
\text { included both } \\
\text { ever smokers } \\
\text { (more than } 100 \\
\text { cigarettes in their } \\
\text { lifetime) and } \\
\text { heavy smokers } \\
\text { (individuals } \\
\text { who consumed } \\
\text { more than } 25 \\
\text { cigarettes } \\
\text { per day) }\end{array}$ & $\begin{array}{c}\text { 1980-1982 } \\
\text { sample: } 4,051 \\
\text { participants, both } \\
\text { sex, aged } 25-74 \\
\text { years; } 1985-1987 \\
\text { sample: } 5,733 \\
\text { participants, both } \\
\text { sex, aged } 25-74 \\
\text { years; } 1990-1992 \\
\text { sample: } 5,994 \\
\text { participants, both } \\
\text { sex, aged } 25-74 \\
\text { years }\end{array}$ & & $\begin{array}{c}\text { 1980-1982 sample: } \\
\text { 65-74 years } \\
\text { (M: 19.4\%/W: 21\%); } \\
\text { 1985-1987 sample: } \\
\text { 65-74 years } \\
\text { (M: 15.4\%/W: } 18.3 \%) ; \\
\text { 1990-1992 sample: } \\
\text { 65-74 years } \\
\text { (M: } 13.8 \% / \mathrm{W}: 16.3 \%)\end{array}$ \\
\hline $\begin{array}{l}\text { Balabanova } \\
\text { et al. } 27 \text { (1998) }\end{array}$ & $\begin{array}{c}\text { Questionnaire } \\
\text { survey }\end{array}$ & $\begin{array}{l}\text { Bulgaria: } \\
\text { household } \\
\text { interview }\end{array}$ & No & $\begin{array}{l}\text { Smokers: those } \\
\text { smoking every } \\
\text { day }\end{array}$ & $\begin{array}{c}1,550 \\
\text { participants, both } \\
\text { sex, aged } \geq 18 \\
\text { years }\end{array}$ & $\begin{array}{c}482 \\
\text { participants } \\
\text { aged } \geq 60 \\
\text { years }\end{array}$ & $\begin{array}{c}60-69 \text { years }(\mathrm{M}: \\
15.5 \% / \mathrm{W}: 1.5 \%), \geq 70 \\
\text { years (M: } 6.2 \% / \mathrm{W}: 0 \%)\end{array}$ \\
\hline $\begin{array}{l}\text { Gilliland et al. } 16 \\
\text { (1998) }\end{array}$ & $\begin{array}{c}\text { Questionnaire } \\
\text { survey }\end{array}$ & $\begin{array}{l}\text { New Mexico } \\
\text { (USA): data } \\
\text { adapted from } \\
\text { the BRFSS for } \\
\text { use with the } \\
\text { American Indian } \\
\text { population } \\
\text { (AlBFRS) }\end{array}$ & $\begin{array}{l}\text { No } \\
\text { information } \\
\text { about proxy } \\
\text { interviews }\end{array}$ & $\begin{array}{l}\text { Current smokers: } \\
\text { ever smokers } \\
\text { (those who } \\
\text { smoked at least } \\
100 \text { cigarettes } \\
\text { in their lifetime) } \\
\text { who were current } \\
\text { smokers at the } \\
\text { time of the } \\
\text { survey }\end{array}$ & $\begin{array}{c}1,275 \\
\text { participants, both } \\
\text { sex, aged } \geq 18 \\
\text { years }\end{array}$ & $\begin{array}{c}80 \text { participants } \\
\text { aged } \geq 65 \\
\text { years }\end{array}$ & $\begin{array}{c}\geq 65 \text { years }(15.3 \% \\
\text { both sex })\end{array}$ \\
\hline $\begin{array}{l}\text { Haidinger } \\
\text { et al. } 50 \text { (1998) }\end{array}$ & $\begin{array}{c}\text { Questionnaire } \\
\text { survey }\end{array}$ & $\begin{array}{c}\text { Austria: } \\
\text { household } \\
\text { interview }\end{array}$ & No & $\begin{array}{l}\text { Current smokers: } \\
\text { included both } \\
\text { regular smokers } \\
\text { (who at the time } \\
\text { of the interview } \\
\text { smoked at least } 1 \\
\text { cigarette per day) } \\
\text { and occasional } \\
\text { smokers (who } \\
\text { at the time of } \\
\text { interview did not } \\
\text { smoke every day) }\end{array}$ & $\begin{array}{c}2,073 \\
\text { participants, } \\
\text { both sex, aged } \geq \\
15 \text { years }\end{array}$ & $\begin{array}{c}289 \\
\text { participants } \\
\text { aged } \geq 65 \\
\text { years (M: } \\
\text { 103/W: 186) }\end{array}$ & $\begin{array}{c}\geq 65 \text { years } \\
\text { (M: } 27.2 \% / \mathrm{W}: 9.7 \%)\end{array}$ \\
\hline
\end{tabular}

(continues) 
Table 1 (continued)

\begin{tabular}{|c|c|c|c|c|c|c|c|}
\hline Author (year) & Methods & Setting & $\begin{array}{c}\text { Proxy } \\
\text { interviews }\end{array}$ & $\begin{array}{c}\text { Definition of } \\
\text { smoking status }\end{array}$ & Participants & $\begin{array}{c}\text { Elderly } \\
\text { participants }\end{array}$ & $\begin{array}{l}\text { Prevalence of } \\
\text { tobacco use }\end{array}$ \\
\hline $\begin{array}{l}\text { Hill et al. } 54 \\
\text { (1998) }\end{array}$ & $\begin{array}{l}\text { Questionnaire } \\
\text { survey }\end{array}$ & $\begin{array}{l}\text { Australia: } \\
\text { household } \\
\text { interview }\end{array}$ & No & $\begin{array}{c}\text { Current smoker: } \\
\text { cigarettes only, } \\
\text { cigarettes plus } \\
\text { cigars nor pipes, } \\
\text { cigars only } \\
\text { (ex-cigarettes), } \\
\text { pipes only } \\
\text { (ex-cigarettes), } \\
\text { cigars only (never } \\
\text { cigarettes), or } \\
\text { pipes only (never } \\
\text { cigarettes) }\end{array}$ & $\begin{array}{c}\text { Aged } \geq 16 \text { years } \\
\begin{array}{c}\text { (M: } 2,819 / \mathrm{W}: \\
2,880)\end{array}\end{array}$ & $\begin{array}{c}369 \text { men aged } \\
60-69 \text { years, } \\
332 \text { men aged } \\
\geq 70 \text { years, } 374 \\
\text { women aged } \\
60-69 \text { years, } \\
339 \text { women } \\
\text { aged } \geq 70 \\
\text { years }\end{array}$ & $\begin{array}{c}\text { 60-69 years } \\
\text { (M: 18.2\%/W: } 14.7 \%), \\
\geq 70 \text { years } \\
\text { (M: } 14.2 \% / \mathrm{W}: 8 \%)\end{array}$ \\
\hline $\begin{array}{l}\text { Idris et al. } 55 \\
\text { (1998) }\end{array}$ & $\begin{array}{c}\text { Questionnaire } \\
\text { survey }\end{array}$ & $\begin{array}{l}\text { Nile State } \\
\text { (Sudan): } \\
\text { household } \\
\text { interview }\end{array}$ & No & $\begin{array}{c}\text { Cigarette } \\
\text { smoking or } \\
\text { toombak use: } \\
\text { self-report daily } \\
\text { use of these } \\
\text { products }\end{array}$ & $\begin{array}{c}21,594 \\
\text { participants, both } \\
\text { sex, aged } \geq 4 \\
\text { years }\end{array}$ & $\begin{array}{l}587 \text { aged } 60- \\
69 \text { years, } 336 \\
\text { aged } 70-79 \\
\text { years }\end{array}$ & $\begin{array}{l}60-69 \text { years (M: } 16.9 \%) ; \\
70-79 \text { years (M: } 15.5 \%) ; \\
\text { women prevalence not } \\
\text { reported (was too low) }\end{array}$ \\
\hline $\begin{array}{l}\text { McKee et al. } 39 \\
\text { (1998) }\end{array}$ & $\begin{array}{c}\text { Questionnaire } \\
\text { survey }\end{array}$ & $\begin{array}{l}\text { Russia: } \\
\text { household } \\
\text { interview }\end{array}$ & No & $\begin{array}{l}\text { Current smokers: } \\
\text { subjects who } \\
\text { gave a positive } \\
\text { reply to the } \\
\text { questions: "Do } \\
\text { you smoke } \\
\text { now?" }\end{array}$ & $\begin{array}{l}1,599 \\
\text { participants, both } \\
\text { sex, aged } \geq 18 \\
\text { years }\end{array}$ & $\begin{array}{l}221 \\
\text { participants } \\
\text { aged } \geq 65 \\
\text { years }\end{array}$ & $\begin{array}{c}\geq 65 \text { years } \\
\text { (M: } 41 \% / \mathrm{W}: 5 \%)\end{array}$ \\
\hline $\begin{array}{l}\text { Osler et al. } 29 \\
\text { (1998) }\end{array}$ & $\begin{array}{c}\text { Questionnaire } \\
\text { survey }\end{array}$ & $\begin{array}{l}\text { Copenhagen } \\
\text { (Denmark): self- } \\
\text { administered } \\
\text { questionnaire }\end{array}$ & No & $\begin{array}{l}\text { Smokers: subjects } \\
\text { who smoke daily } \\
\text { or sometimes } \\
\text { at the time of } \\
\text { examination; } \\
\text { heavy smokers: } \\
\text { smokers } \\
\text { with a daily } \\
\text { consumption of } \\
\text { more than } 14 \mathrm{~g} \text { of } \\
\text { tobacco }\end{array}$ & $\begin{array}{l}33,655 \\
\text { participants, both } \\
\text { sex, aged } \geq 30 \\
\text { years }\end{array}$ & $\begin{array}{l}15,478 \text { aged } \\
50-69 \text { years; } \\
2,408 \text { aged } \geq \\
70 \text { years }\end{array}$ & $\begin{array}{l}\text { 50-69 years (current } \\
\text { smokers: } 63 \% \text { both } \\
\text { sex/heavy smokers: } \\
27 \% \text { both sex), } \geq 70 \\
\text { years (current smokers: } \\
48 \% \text { both sex/heavy } \\
\text { smokers: } 9 \% \text { both sex) }\end{array}$ \\
\hline $\begin{array}{l}\text { Pagano et al. } 25 \\
\text { (1998) }\end{array}$ & $\begin{array}{l}\text { Questionnaire } \\
\text { survey }\end{array}$ & $\begin{array}{l}\text { Italy: household } \\
\text { interview }\end{array}$ & $\begin{array}{l}\text { Yes - proxy } \\
\text { interviews } \\
\text { represented } \\
25 \% \text { of total } \\
\text { sample }\end{array}$ & $\begin{array}{c}\text { Current smoker: } \\
\text { heavy current } \\
\text { smoker (those } \\
\text { currently smoking } \\
\geq 15 \text { cigarettes } \\
\text { per day) }\end{array}$ & $\begin{array}{c}50,585 \\
\text { participants, both } \\
\text { sex, aged } \geq 15 \\
\text { years }\end{array}$ & $\begin{array}{c}2,553 \text { men } \\
\text { aged } 65-74 \\
\text { years; } 1,240 \\
\text { men aged } \geq \\
75 \text { years; } 3,107 \\
\text { women aged } \\
65-74 \text { years; } \\
2,034 \text { women } \\
\text { aged } \geq 75 \\
\text { years }\end{array}$ & $\begin{array}{c}\text { 65-74 years (current } \\
\text { smokers: M: 24.3\%/W: } \\
\text { 6.9\%; heavy smokers: } \\
\text { M: 8.8\%/W: } 1.4 \% \text { ); } 75 \\
\text { years (current smokers: } \\
\text { M: } 14.1 \% / \mathrm{W}: 3.1 \% \text {; } \\
\text { heavy smokers: M: } \\
\text { 3.1\%/W: } 0.4 \% \text { ) }\end{array}$ \\
\hline
\end{tabular}

(continues) 
Table 1 (continued)

\begin{tabular}{|c|c|c|c|c|c|c|c|}
\hline Author (year) & Methods & Setting & $\begin{array}{c}\text { Proxy } \\
\text { interviews }\end{array}$ & $\begin{array}{c}\text { Definition of } \\
\text { smoking status }\end{array}$ & Participants & $\begin{array}{c}\text { Elderly } \\
\text { participants }\end{array}$ & $\begin{array}{l}\text { Prevalence of } \\
\text { tobacco use }\end{array}$ \\
\hline $\begin{array}{l}\text { Smedslund \& } \\
\text { Ahn } 20 \text { (1998) }\end{array}$ & $\begin{array}{c}\text { Questionnaire } \\
\text { survey }\end{array}$ & $\begin{array}{l}\text { Stanford, } \\
\text { California (USA): } \\
\text { interview held } \\
\text { in a health } \\
\text { center/Bergen } \\
\text { and Trondheim } \\
\text { (Norway): } \\
\text { questionnaires } \\
\text { were sent to } \\
\text { respondents } \\
\text { homes }\end{array}$ & $\begin{array}{c}\text { No } \\
\text { information } \\
\text { about proxy } \\
\text { interviews. } \\
\text { The } \\
\text { questionnaire } \\
\text { was sent to } \\
\text { the houses }\end{array}$ & Current smokers & $\begin{array}{c}\text { California } \\
\text { sample: } 2,189 \\
\text { participants, both } \\
\text { sex, aged 18-74 } \\
\text { years; Norway } \\
\text { sample: 5,014 } \\
\text { participants, both } \\
\text { sex, aged 19-92 } \\
\text { years }\end{array}$ & & $\begin{array}{l}\text { California sample: } 60- \\
69 \text { years (M: 24\%/W: } \\
\text { 15\%), 70-79 years (M: } \\
\text { 11\%/W: } 15 \% \text { ); Norway } \\
\text { sample: } 60-69 \text { years (M: } \\
\text { 23\%/W: } 20 \%), 70-79 \\
\text { years (M: } 19 \% / \mathrm{W}: 17 \%)\end{array}$ \\
\hline $\begin{array}{l}\text { Wiecha et al. } 12 \\
\text { (1998) }\end{array}$ & $\begin{array}{c}\text { Questionnaire } \\
\text { survey }\end{array}$ & $\begin{array}{l}\text { Massachusetts } \\
\text { (USA): telephone } \\
\text { interview }\end{array}$ & No & $\begin{array}{l}\text { Current smokers: } \\
\text { those who had } \\
\text { smoked at least } \\
100 \text { cigarettes } \\
\text { and who had } \\
\text { smoked any part } \\
\text { of a cigarette in } \\
\text { the past } 30 \text { days }\end{array}$ & $\begin{array}{l}774 \text { Vietnamese } \\
\text { men residents in } \\
\text { Massachusetts } \\
\text { aged } \geq 18 \text { years }\end{array}$ & $\begin{array}{c}52 \text { participants } \\
\text { aged } \geq 60 \\
\text { years }\end{array}$ & $\geq 60$ years (M: $36.5 \%)$ \\
\hline $\begin{array}{l}\text { Nebot et al. } 28 \\
\text { (1996) }\end{array}$ & $\begin{array}{c}\text { Questionnaire } \\
\text { survey }\end{array}$ & $\begin{array}{l}\text { Spain: household } \\
\text { interview }\end{array}$ & No & $\begin{array}{l}\text { Smokers: } \\
\text { smoking tobacco } \\
\text { products at } \\
\text { the time of the } \\
\text { survey }\end{array}$ & $\begin{array}{c}1983 \text { sample: } \\
3,134 \\
\text { participants, } \\
\text { both sex, aged } \\
\geq 15 \text { years; } 1992 \\
\text { sample: } 5,004 \\
\text { participants, both } \\
\text { sex, aged } \geq 15 \\
\text { years }\end{array}$ & & $\begin{array}{c}1983 \text { sample: } \geq 65 \\
\text { years (M: } 40.1 \% / \mathrm{W}: \\
4.7 \%), 1992 \text { sample: } \geq \\
65 \text { years (M: } 26.4 \% / \mathrm{W}: \\
3.6 \%)\end{array}$ \\
\hline $\begin{array}{l}\text { Resnicow } \\
\text { et al. } 30 \text { (1996) }\end{array}$ & $\begin{array}{c}\text { Questionnaire } \\
\text { survey }\end{array}$ & $\begin{array}{l}\text { Harlem, New } \\
\text { York (USA): self- } \\
\text { administered } \\
\text { questionnaire }\end{array}$ & No & $\begin{array}{l}\text { Smokers: } \\
\text { smoking tobacco } \\
\text { products at } \\
\text { the time of the } \\
\text { survey }\end{array}$ & $\begin{array}{c}7,761 \\
\text { participants, both } \\
\text { sex, aged } \geq 18 \\
\text { years }\end{array}$ & & $\begin{array}{c}\geq 60 \text { years }(\mathrm{M}: \\
33.9 \% / \mathrm{W}: 24.8 \%)\end{array}$ \\
\hline $\begin{array}{l}\text { Shopland } \\
\text { et al. } 26 \text { (1996) }\end{array}$ & $\begin{array}{l}\text { Questionnaire } \\
\text { survey: Current } \\
\text { Population } \\
\text { Survey }\end{array}$ & $\begin{array}{l}\text { USA: household } \\
\text { interview }\end{array}$ & Yes & $\begin{array}{l}\text { Current smokers: } \\
\text { if they had ever } \\
\text { smoked at least } \\
100 \text { cigarettes } \\
\text { in their entire } \\
\text { lifetime and if } \\
\text { they currently } \\
\text { smoked } \\
\text { cigarettes every } \\
\text { day or some days }\end{array}$ & $\begin{array}{c}266,988 \\
\text { participants, both } \\
\text { sex, aged } \geq 20 \\
\text { years }\end{array}$ & & $\begin{array}{c}60-69 \text { years }(\mathrm{M}: \\
20.5 \% / \mathrm{W}: 17.3 \%), 70- \\
79 \text { years (M: } 11 \% / \mathrm{W}: \\
8.8 \%)\end{array}$ \\
\hline $\begin{array}{l}\text { Ganiwijaya } \\
\text { et al. } 21 \text { (1995) }\end{array}$ & $\begin{array}{c}\text { Questionnaire } \\
\text { survey }\end{array}$ & $\begin{array}{l}\text { West Java } \\
\text { (Indonesia): } \\
\text { household } \\
\text { interview }\end{array}$ & Yes & $\begin{array}{l}\text { Current smokers: } \\
\text { defined as those } \\
\text { who had smoked } \\
\text { in the past month }\end{array}$ & $\begin{array}{c}13,863 \\
\text { participants, both } \\
\text { sex, aged } 25-74 \\
\text { years }\end{array}$ & $\begin{array}{l}\quad 834 \\
\text { participants } \\
\text { aged } 65-74 \\
\text { years }\end{array}$ & $\begin{array}{l}65-74 \text { years }(\mathrm{M}: \\
84.5 \% / \mathrm{W}: 6.8 \%)\end{array}$ \\
\hline
\end{tabular}

(continues) 


\begin{tabular}{|c|c|c|c|c|c|c|c|}
\hline Author (year) & Methods & Setting & $\begin{array}{c}\text { Proxy } \\
\text { interviews }\end{array}$ & $\begin{array}{c}\text { Definition of } \\
\text { smoking status }\end{array}$ & Participants & $\begin{array}{c}\text { Elderly } \\
\text { participants }\end{array}$ & $\begin{array}{l}\text { Prevalence of } \\
\text { tobacco use }\end{array}$ \\
\hline $\begin{array}{l}\text { Gong et al. } 43 \\
\text { (1995) }\end{array}$ & $\begin{array}{c}\text { Questionnaire } \\
\text { survey }\end{array}$ & $\begin{array}{c}\text { Minhang } \\
\text { District (China): } \\
\text { household } \\
\text { interview }\end{array}$ & No & $\begin{array}{l}\text { Current smokers: } \\
\text { persons who } \\
\text { had smoked } \\
\text { more than } 100 \\
\text { cigarettes in their } \\
\text { lifetimes and } \\
\text { who were still } \\
\text { smoking daily at } \\
\text { the time of the } \\
\text { interview }\end{array}$ & $\begin{array}{c}7,016 \\
\text { participants, both } \\
\text { sex, aged } \geq 15 \\
\text { years }\end{array}$ & $\begin{array}{l}780 \text { aged } 60- \\
69,453 \text { aged } \\
\quad \geq 70\end{array}$ & $\begin{array}{c}\text { 60-69 years (M: } \\
56.6 \% / \mathrm{W}: 4.3 \% / \text { both } \\
\text { sex: } 30.1 \%), \geq 70 \text { years } \\
\text { (M: } 45.3 \% / \mathrm{W}: 4.3 \% / \\
\text { both sex: } 19.9 \%)\end{array}$ \\
\hline $\begin{array}{l}\text { Gutiérrez et al. } 32 \\
\text { (1995) }\end{array}$ & $\begin{array}{c}\text { Questionnaire } \\
\text { survey }\end{array}$ & $\begin{array}{l}\text { Valparaíso } \\
\text { and Viña del } \\
\text { Mar (Chile): } \\
\text { household } \\
\text { interview }\end{array}$ & No & $\begin{array}{l}\text { Smoker: smoking } \\
\text { one or more } \\
\text { cigarettes weekly } \\
\text { for } 6 \text { months } \\
\text { or more before } \\
\text { the survey }\end{array}$ & $\begin{array}{c}1,959 \\
\text { participants, both } \\
\text { sex, aged } \geq 8 \\
\text { years }\end{array}$ & & $\begin{array}{c}\geq 60 \text { years }(\mathrm{M}: \\
27.4 \% / \mathrm{W}: 8.1 \%)\end{array}$ \\
\hline $\begin{array}{l}\text { La Vecchia } \\
\text { et al. } 23 \text { (1994) }\end{array}$ & $\begin{array}{c}\text { Questionnaire } \\
\text { survey }\end{array}$ & $\begin{array}{l}\text { Italy: household } \\
\text { interview }\end{array}$ & $\begin{array}{l}\text { Yes - proxy } \\
\text { interviews } \\
\text { represented } \\
8 \% \text { of total } \\
\text { sample }\end{array}$ & Current smoker & $\begin{array}{c}55,989 \\
\text { participants, both } \\
\text { sex, aged } \geq 15 \\
\text { years }\end{array}$ & $\begin{array}{c}5,332 \text { aged } 65- \\
74 ; 2,972 \text { aged } \\
75-84 ; 572 \\
\text { aged } \geq 85\end{array}$ & $\begin{array}{c}65-74 \text { years }(\mathrm{M}: 29 \% / \mathrm{W}: \\
6.7 \%), 75-84 \text { years } \\
\text { (M: } 18.9 \% / \mathrm{W}: 2.5 \%), \geq \\
85 \text { years (M: } 17.1 \% / \mathrm{W}: \\
0.7 \%)\end{array}$ \\
\hline $\begin{array}{l}\text { Woodward } \\
\text { et al. } 49 \text { (1994) }\end{array}$ & $\begin{array}{c}\text { Questionnaire } \\
\text { survey }\end{array}$ & $\begin{array}{c}\text { Tonga: } \\
\text { household } \\
\text { interview }\end{array}$ & No & $\begin{array}{l}\text { Current } \\
\text { smokers: those } \\
\text { respondents who } \\
\text { reported that } \\
\text { they had smoked } \\
\text { in the three } \\
\text { months prior to } \\
\text { the survey }\end{array}$ & $\begin{array}{l}4,065 \\
\text { participants, both } \\
\text { sex, aged } \geq 20 \\
\text { years }\end{array}$ & $\begin{array}{l}775 \\
\text { participants } \\
\text { aged } \geq 60 \\
\text { years }\end{array}$ & $\begin{array}{c}\text { 60-64 years } \\
\text { (M: 66.4\%/W: } 16.3 \%), \\
\text { 65-69 years (M: } \\
\text { 54.5\%/W: } 26.1 \%), 70- \\
74 \text { years (M: } 60.9 \% / \mathrm{W}: \\
\text { 15.5\%), } 75-79 \text { years } \\
\text { (M: 43.4\%/W: } 14.6 \%), \geq \\
80 \text { years (M: } 43.9 \% / \mathrm{W}: \\
25.9 \%)\end{array}$ \\
\hline $\begin{array}{l}\text { Lolio et al. } 37 \\
\text { (1993) }\end{array}$ & $\begin{array}{c}\text { Questionnaire } \\
\text { survey }\end{array}$ & $\begin{array}{l}\text { Araraquara } \\
\text { (Brazil): } \\
\text { household } \\
\text { interview }\end{array}$ & No & $\begin{array}{l}\text { Smoker: cigarette } \\
\text { and/or piper and/ } \\
\text { or cigar for } 6 \\
\text { months or more, } \\
\text { every day, up to } \\
\text { the moment } \\
\text { of the } \\
\text { interview }\end{array}$ & $\begin{array}{l}1,199 \\
\text { participants, both } \\
\text { sex, aged 15-74 } \\
\text { years }\end{array}$ & & $\begin{array}{c}65-74 \text { years } \\
\text { (M: 58\%/W: } 6.6 \%)\end{array}$ \\
\hline
\end{tabular}

(continues) 
Table 1 (continued)

\begin{tabular}{|c|c|c|c|c|c|c|c|}
\hline Author (year) & Methods & Setting & $\begin{array}{c}\text { Proxy } \\
\text { interviews }\end{array}$ & $\begin{array}{l}\text { Definition of } \\
\text { smoking status }\end{array}$ & Participants & $\begin{array}{c}\text { Elderly } \\
\text { participants }\end{array}$ & $\begin{array}{l}\text { Prevalence of } \\
\text { tobacco use }\end{array}$ \\
\hline $\begin{array}{l}\text { Maxwell \& } \\
\text { Hirdes } 18 \text { (1993) }\end{array}$ & $\begin{array}{c}\text { Questionnaire } \\
\text { survey }\end{array}$ & $\begin{array}{l}\text { Canada - 1985: } \\
\text { household } \\
\text { survey; 1986: } \\
\text { telephone } \\
\text { interview; 1989: } \\
\text { telephone } \\
\text { interview }\end{array}$ & $\begin{array}{l}1985 \text { - No; } \\
1986 \text { - Yes; } \\
1989 \text { - Yes }\end{array}$ & $\begin{array}{l}\text { Current smokers: } \\
\text { those reporting } \\
\text { that they smoked } \\
\text { cigarettes daily } \\
\text { at the time of the } \\
\text { survey }\end{array}$ & $\begin{array}{c}1985 \text { sample: } \\
11,200 \\
\text { participants, } \\
\text { both sex, aged } \\
\geq 15 \text { years; } 1986 \\
\text { sample: } 30,799 \\
\text { participants, } \\
\text { both sex, aged } \\
\geq 15 \text { years; } 1989 \\
\text { sample: } 11,634 \\
\text { participants, both } \\
\text { sex, aged } \geq 15 \\
\text { years }\end{array}$ & $\begin{array}{c}1985 \text { sample: } \\
3,130 \\
\text { participants, } \\
\text { both sex, } \\
\text { aged } \geq 65 \\
\text { years; } 1986 \\
\text { sample: } 4,135 \\
\text { participants, } \\
\text { both sex, } \\
\text { aged } \geq 65 \\
\text { years; } 1989 \\
\text { sample: } 1,758 \\
\text { participants, } \\
\text { both sex, aged } \\
\geq 65 \text { years }\end{array}$ & $\begin{array}{c}1985 \text { sample: } 65-69 \\
\text { years (M: } 28.4 \% / \mathrm{W}: \\
\text { 20\%), 70-74 years (M: } \\
23.3 \% / \mathrm{W}: 18.1 \%), 75- \\
79 \text { years (M: } 21.4 \% / \mathrm{W}: \\
13.1 \%), \geq 80 \text { years (M: } \\
\text { 13.2\%/W: } 4.6 \%) ; 1986 \\
\text { sample: 65-69 years } \\
\text { (M: 22.9\%/W: } 16.5 \%), \geq \\
70 \text { years (M: } 16.5 \% / \mathrm{W}: \\
8.7 \%) ; 1989 \text { sample: 65- } \\
69 \text { years (M: } 23.9 \% / \mathrm{W}: \\
19.4 \%), 70-74 \text { years } \\
\text { (M: } 23.3 \% / \mathrm{W}: 19 \%), \\
75-79 \text { years (M: } 16 \% / \mathrm{W}: \\
15.1 \%), \geq 80 \text { years (M: } \\
13.5 \% / \mathrm{W}: 9.4 \%)\end{array}$ \\
\hline $\begin{array}{l}\text { Hamadeh et al. } \\
22 \text { (1992) }\end{array}$ & $\begin{array}{c}\text { Questionnaire } \\
\text { survey }\end{array}$ & $\begin{array}{l}\text { Bahrain: } \\
\text { household } \\
\text { interview }\end{array}$ & $\begin{array}{l}\text { Yes - the } \\
\text { questionnaire } \\
\text { was } \\
\text { presented } \\
\text { to the } \\
\text { head of the } \\
\text { household } \\
\text { or the eldest } \\
\text { person in } \\
\text { the presence } \\
\text { of adult } \\
\text { members of } \\
\text { the family }\end{array}$ & $\begin{array}{l}\text { Cigarette } \\
\text { smokers: regular } \\
\text { light smokers } \\
\text { (those who } \\
\text { smoked less than } \\
1 \text { packet a day), } \\
\text { regular heavy } \\
\text { smokers (those } \\
\text { who smoked } 1 \\
\text { packet or more } \\
\text { daily), occasional } \\
\text { smokers (those } \\
\text { who smoked less } \\
\text { than } 1 \text { cigarette a } \\
\text { day). Waterpipe } \\
\text { smokers: regular } \\
\text { smokers (those } \\
\text { who smoked } \\
\text { daily), occasional } \\
\text { smokers }\end{array}$ & $\begin{array}{c}9,282 \\
\text { participants, both } \\
\text { sex, aged } \geq 15 \\
\text { years }\end{array}$ & & $\begin{array}{c}60-69 \text { years }(\mathrm{M}: \\
\text { 40.9\%W: } 16.7 \%),>70 \\
\text { years ( } \mathrm{M}: 28.8 \% / \mathrm{W}: \\
24.8 \%)\end{array}$ \\
\hline
\end{tabular}

BFRSS: Behavioral Risk Factor Surveillance System; H-EPESE: Hispanic Established Populations for Epidemiological Studies of the Elderly; HHANES: Hispanic Health and Nutrition Examination Survey; INCA: Instituto Nacional de Câncer [Brazilian National Cancer Institute]; M: men; NHIS: National Health Interview Survey; USA: United States; USSR: Union of Soviet Socialist Republics; W: women. 


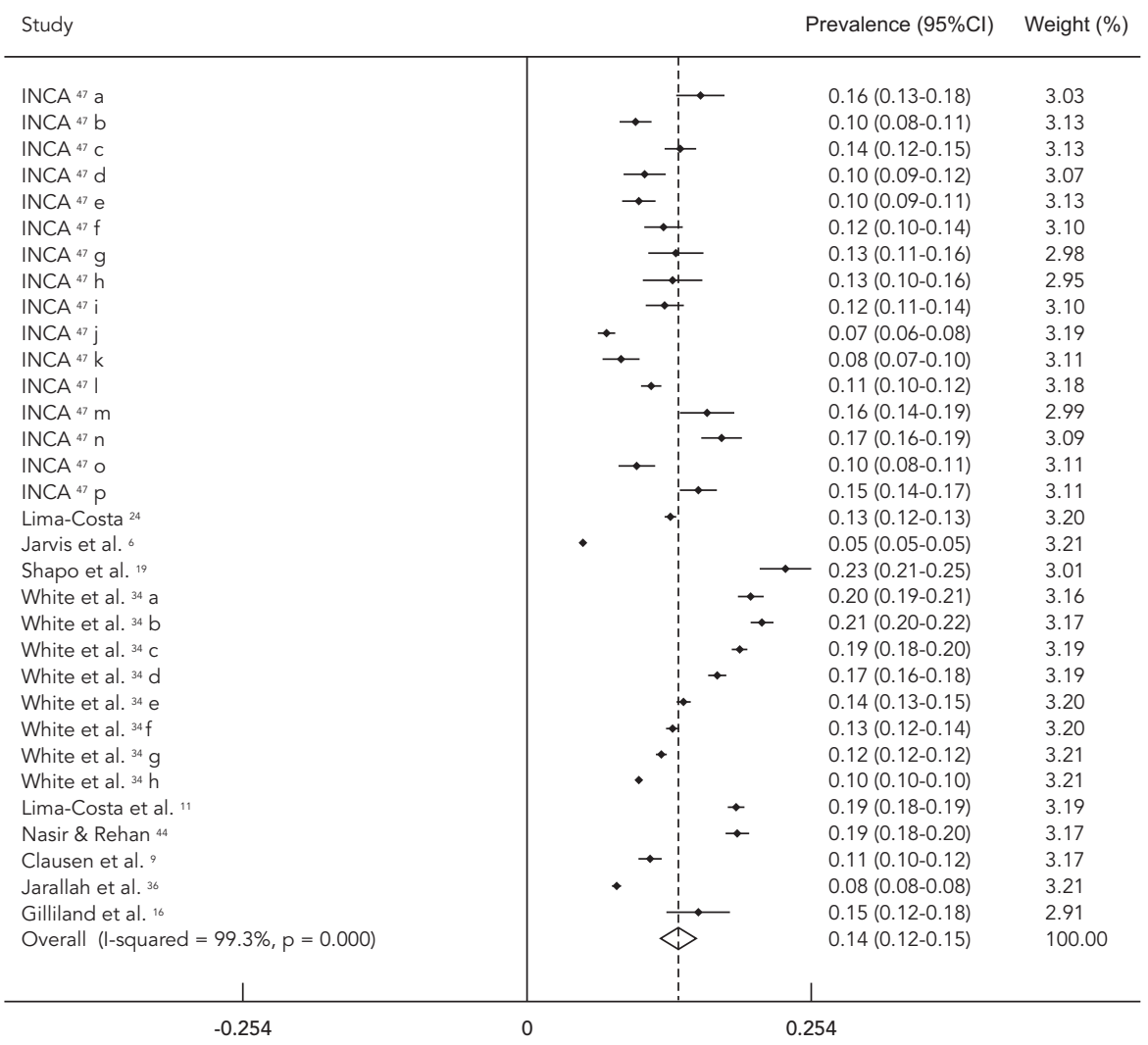

Note: weights are from random effects analysis.

an important heterogeneity of the prevalence rates among the studies.

Aiming to detect which variables might be responsible for that variability we carried out a meta-regression including: smoking definition, method of questionnaire application (household, telephone), country economic status, and continent. Prevalence varied by age group, with a general tendency of decreasing prevalence rates with advancing age. As studies used different age categories, this hypothesis could not be tested. Some surveys considered as elderly those aged 55 -years and older, 60-years and older, 65-years and older and 70-years and older, whereas others used categories of age in more than two groups, 61-65 years, $66-70$ years, $>70$ years; $55-69 ; 60-69$, $\geq 70$; $65-74, \geq 75$; $50-88$ years.

Smoking definition was associated with prevalence variability. Smoking definition according to the Centers for Disease Control and Prevention (CDC) criteria (having smoked at least 100 ciga- rettes in one's lifetime and currently smoke every day or some days) was considered the more accurate characterization 56 . The CDC criteria yielded a prevalence which was $22 \%$ lower in men and $10 \%$ lower in women as compared to less accurate definitions. However, CDC studies showed a higher prevalence than those which did not attempt to define tobacco use among women $(8 \%$ increase). Questionnaire application (telephone/ face-to-face/mailed) also interfered on prevalence rates. Although statistical significance was borderline $(\mathrm{p}=0.08)$, the prevalence rates in men were higher in surveys conducted via telephone calls than in face to face surveys. There was also a trend in enhancing smoking prevalence among women in surveys conducted in a health center, $10 \%$ higher than household face-to-face surveys.

Demographic factors such as continent and country economy status also had a statistically significant impact on smoking prevalence rates among men. High income countries have a $10 \%$ 
Smoking prevalence rates among the elderly (male).

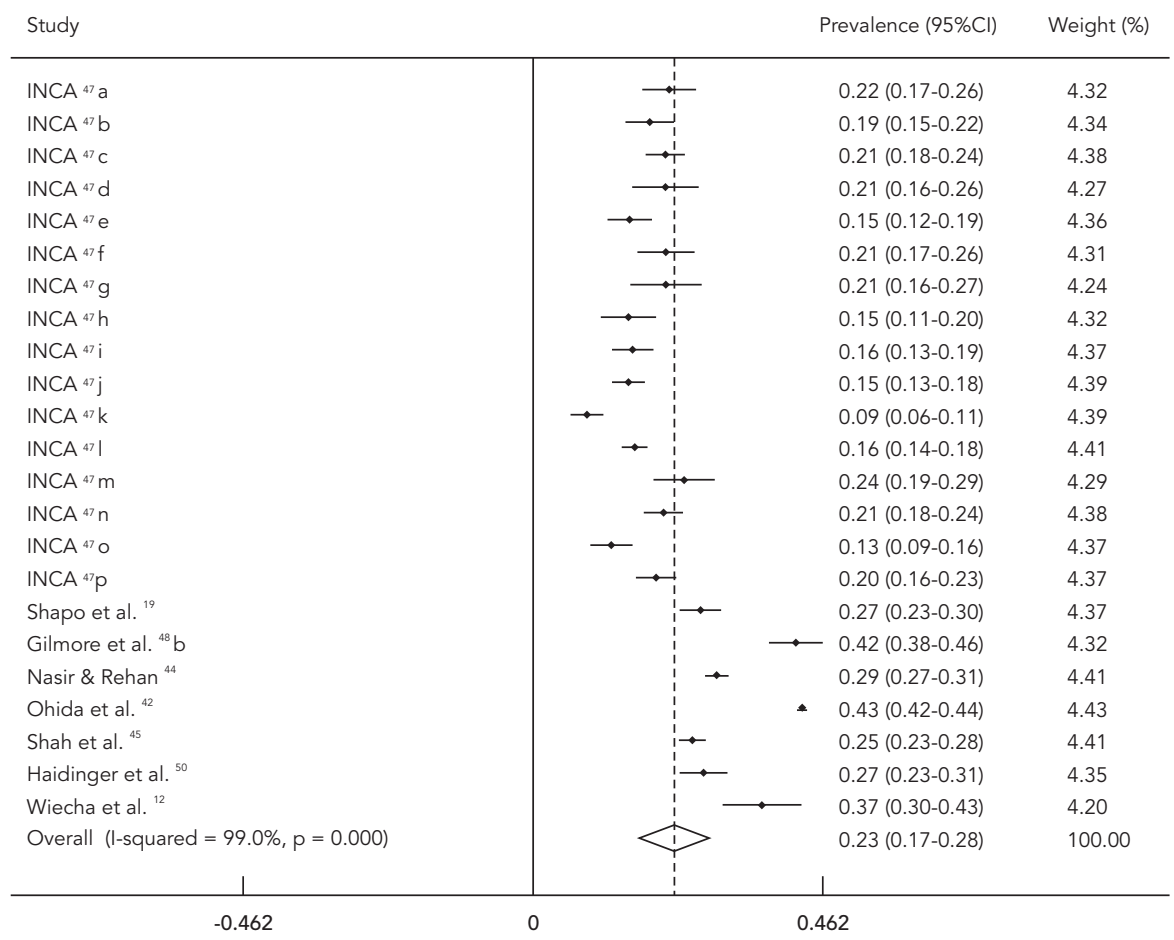

Note: weights are from random effects analysis.

higher prevalence rate than lower middle income countries. Taking Latin America as a reference, there was a $10 \%$ increase in prevalence rates in Asia and, a 19\% increase in North America, whereas Europe showed a 14\% increase (Table 2).

\section{Discussion}

This review identified a large number of surveys on tobacco use, but only three of them were specifically suited to search health and sociodemographic factors in the elderly population. Almost all surveys included elderly in the total adult sample and used questionnaires tailored to the adult population.

The meta-analysis suggests a higher smoking prevalence among men. Gender differences in tobacco use are well known among adults 57, although recent cohorts have shown an increased use in young women 58 . Such estimates imply that in the future tobacco use may increase among elderly women, reproducing the trend observed in young cohorts.
Concerning age groups, the initial assumption of a decreasing prevalence with advanced age could not be evaluated properly in this study due to methodological issues. Despite the fact that there was a trend in decreasing prevalence rates among the oldest elderly, this could not be established because of the variability in definitions of age groups. Lower prevalence among the very oldest could be explained by selective survival probabilities (smokers die earlier) or by birth cohort effect. Cross-sectional studies do not allow for discrimination between these two possibilities.

The observed heterogeneity in prevalence rates among surveys could be partially explained by different smoking definitions, questionnaire application strategies, and country economic status. Prevalence rates were related to the accuracy in measures of tobacco use. Less accurate definitions produced higher prevalence rates, since they included occasional, daily, and heavy smokers without distinction. Nevertheless, there was an unexpected finding of lower prevalence rates related to studies that classified tobacco 


\begin{tabular}{|c|c|c|c|}
\hline Study & & Prevalence $(95 \% \mathrm{Cl})$ & Weight (\%) \\
\hline INCA ${ }^{47} a$ & {$[-$} & $0.11(0.09-0.13)$ & 4.45 \\
\hline INCA ${ }^{47} \mathrm{~b}$ & $\rightarrow$ & $0.04(0.03-0.04)$ & 4.68 \\
\hline INCA ${ }^{47} \mathrm{C}$ & & $0.09(0.07-0.10)$ & 4.62 \\
\hline INCA ${ }^{47} d$ & $\rightarrow$ & $0.04(0.03-0.04)$ & 4.67 \\
\hline INCA ${ }^{47} \mathrm{e}$ & $\rightarrow$ & $0.07(0.05-0.08)$ & 4.61 \\
\hline INCA ${ }^{47} \mathrm{f}$ & $\rightarrow$ & $0.07(0.06-0.09)$ & 4.60 \\
\hline INCA ${ }^{47} \mathrm{~g}$ & $\ldots .1$ & $0.08(0.06-0.10)$ & 4.47 \\
\hline INCA ${ }^{47} \mathrm{~h}$ & & $0.11(0.08-0.14)$ & 4.14 \\
\hline INCA ${ }^{47} \mathrm{i}$ & & $0.10(0.08-0.12)$ & 4.51 \\
\hline INCA ${ }^{47} \mathrm{j}$ & + & $0.03(0.02-0.03)$ & 4.70 \\
\hline INCA ${ }^{47} \mathrm{~K}$ & & $0.08(0.06-0.10)$ & 4.44 \\
\hline$\left|N C A{ }^{47}\right|$ & $\overrightarrow{0}$ & $0.08(0.07-0.09)$ & 4.67 \\
\hline INCA ${ }^{47} \mathrm{~m}$ & & $0.09(0.07-0.11)$ & 4.46 \\
\hline INCA ${ }^{47} \mathrm{n}$ & $\longrightarrow$ & $0.15(0.13-0.17)$ & 4.45 \\
\hline INCA ${ }^{47} \mathrm{O}$ & & $0.08(0.07-0.10)$ & 4.53 \\
\hline INCA ${ }^{47} \mathrm{P}$ & $\rightarrow$ & $0.13(0.11-0.14)$ & 4.52 \\
\hline Shapo et al. ${ }^{19}$ & $\longrightarrow$ & $\rightarrow 0.18(0.15-0.21)$ & 4.21 \\
\hline Gilmore et al. ${ }^{48} \mathrm{~b}$ & - & $0.01(0.01-0.01)$ & 4.71 \\
\hline Nasir \& Rehan ${ }^{44}$ & $\rightarrow$ & $0.05(0.04-0.06)$ & 4.69 \\
\hline Ohida et al. ${ }^{42}$ & $\bullet$ & $0.08(0.07-0.08)$ & 4.71 \\
\hline Shah et al. ${ }^{45}$ & $\rightarrow$ & $0.18(0.17-0.20)$ & 4.52 \\
\hline Haidinger et al. ${ }^{50}$ & 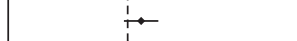 & $0.10(0.08-0.11)$ & 4.62 \\
\hline Overall (I-squared $=99.6 \%, p=0.000$ ) & & $0.09(0.07-0.11)$ & 100.00 \\
\hline 1 & & $\perp$ & \\
\hline-0.211 & 0.2 & 211 & \\
\hline
\end{tabular}

Note: weights are from random effects analysis.

use as "no definition" as compared to those with more accurate definitions. This could be caused by chance as only one study categorized smokers as "no definition", and the reported prevalence was very low $(0.7 \%) 48$.

Several studies show that the methodology of questionnaire application (telephone/face-toface) does not affect smoking prevalence rates among surveys conducted in the community $59,60,61$. However, contrasting results from recent follow-up studies suggest that significant differences regarding socio-demographic variables arise among smokers who gave answers by telephone and by mailing 62,63 . Indeed, this is a very important issue when studying communitydwelling elderly, given that this population usually has hearing, visual, and movement impairments that may complicate proper data collection which use these means.

Albeit not statistically significant, this study found a higher prevalence among surveys carried out in health centers and among those using questionnaires answered by telephone. Cross- sectional surveys in health centers are prone to a selection bias. Elderly people who attend senior citizen centers may be in poorer health conditions 64 and are more likely to be engaged in unhealthy behaviors, possibly explaining enhanced prevalence rates of smoking found in these facilities. Telephone interviews also produced a non significant statistical increase in smoking prevalence, contradicting an initial postulation that elderly subjects would be less suitable to answer telephone interviews and to give proper answers to the questions on tobacco use. Hence, such a result suggests that telephone interviews can be an accurate way of measuring tobacco use among the elderly, since differences found were small, not statistically significant, and were more likely to over- rather than under-estimate prevalence rates.

Information on tobacco use was mostly obtained by self-reporting, whereas proxy responses were allowed in nine surveys. The impact of proxy responses and the validity of self-reported smoking on estimates of tobacco use among the 


\section{Table 2}

Meta-regression investigating variables associated with heterogeneity among men and women.

\begin{tabular}{|c|c|c|}
\hline Variable & Coefficient & $95 \% \mathrm{Cl}$ \\
\hline \multicolumn{3}{|l|}{ Men } \\
\hline \multicolumn{3}{|l|}{ Tobacco definition } \\
\hline WHO/CDC criteria & Reference & \\
\hline Current & 0.06 & $-0.05 ; 0.18$ \\
\hline No definition & 0.22 * & $0.10 ; 0.34$ \\
\hline Occasional smokers & 0.07 & $-0.05 ; 0.19$ \\
\hline Hardcore + heavy smokers & - & - \\
\hline \multicolumn{3}{|l|}{ Interview methodology } \\
\hline Face-to-face & Reference & \\
\hline Telephone & $0.15^{\star *}$ & $-0.02 ; 0.33$ \\
\hline Health center & 0.58 & $-0.08 ; 0.19$ \\
\hline Mailed questionnaires & - & - \\
\hline \multicolumn{3}{|l|}{ Country economic status } \\
\hline High income & Reference & \\
\hline Lower middle income & $-0.10 * \star \star *$ & $-0.21 ; 0.01$ \\
\hline Upper middle income & - & - \\
\hline Low income & - & - \\
\hline \multicolumn{3}{|l|}{ Continent } \\
\hline Latin America & Reference & \\
\hline Asia & 0.10 * & $0.06 ; 0.14$ \\
\hline North America & 0.19 * & $0.05 ; 0.32$ \\
\hline Europe & 0.14 * & $0.08 ; 0.19$ \\
\hline Oceania & - & - \\
\hline Africa & - & - \\
\hline \multicolumn{3}{|l|}{ Women } \\
\hline \multicolumn{3}{|l|}{ Tobacco definition } \\
\hline WHO/CDC criteria & Reference & \\
\hline Current & $0.1 \#$ & $-0.003 ; 0.2$ \\
\hline No definition & $-0.08 \# \#$ & $-0.14 ;-0.01$ \\
\hline Occasional smokers & 0.01 & $-0.05 ; 0.09$ \\
\hline Hardcore + heavy smokers & - & - \\
\hline \multicolumn{3}{|l|}{ Interview methodology } \\
\hline Face-to-face & Reference & \\
\hline Health center & $0.1 \# \#$ & $-0.007 ; 0.21$ \\
\hline Mailed questionnaires & - & - \\
\hline Telephone & - & - \\
\hline
\end{tabular}

${ }^{*} p<0.001$;

${ }^{\star *} p=0.08$.

$* * * p=0.09 ;$

$\# p=0.06$;

$\# p<0.05$;

$\# \# \mathrm{p}=0.07$

elderly remain uncertain. Proxy responses might under-estimate smoking prevalence. On the other hand, self-reported responses are considered a safe and economic measure of tobacco use in population studies 65 . The present results suggest that self-reporting screening is also a safe measure of tobacco use among the elderly, since there was no difference in prevalence rates of proxy responses as compared to those obtained by the former method.

There is also evidence that elderly people living in countries with better socio-economic conditions have higher prevalence rates of tobacco use than those living in under-developed coun- 
tries. Countries and continents which include more developed nations were the most likely regions to present higher prevalence rates. This result contrasts with recent findings suggesting that low socio-economic conditions are related with tobacco use, both at individual 13,33,37,38,46,54,66 and domiciliary level 66,67. A possible explanation for such a finding is that rates of cigarette smoking in developed countries have decreased mainly among adults. Tobacco control activities usually focus on young adults, and as a result elderly people living in those countries may not be exposed to those educational programs. Tobacco use among future elderly cohorts may decrease following the trend observed in young cohorts.

A range of different factors precluded the conclusions on frequency of tobacco use among the elderly to be drawn as precisely as we wanted. These included variability in elderly sample size among surveys, study quality and design, heterogeneity in data collection such as differences in smoking definitions and inclusion of regional tobacco products, classification of elderly according to age ranges, and limitations from metaanalysis methodology.

Limitations of this study include those common to all meta-analysis research, since this procedure can be affected by the methodological quality of the original studies. Furthermore, sample size and response rates can represent a selection bias when calculating smoking prevalence, since smokers are less prone to answer questions on tobacco use 63. In almost all selected surveys, response rates in the elderly had not been described routinely. Also, not all the countries had elderly samples equally represented and cultural aspects that may affect tobacco use definitions and the methodology of data gathering might vary between regions and countries studied, becoming a confounder in the causal relationship between country and smoking prevalence. Finally, we must consider that other databases such as Embase, Scopus, PsychLit books, Masters or $\mathrm{PhD}$ theses were not included in our research strategy.

Thus, the conclusions on this meta-analysis can not be generalized as representative of a pattern of smoking behavior among elderly subjects worldwide.

\section{Conclusion}

Tobacco use among the elderly is an important and potentially preventable health problem. However, few epidemiological studies have assessed rates of tobacco consumption among the aged so far. Additional evidence regarding elderly samples, using questionnaires tailored to investigate their main health problems and socio-demographic distress factors is required. The methodology applied may impact on the results, more precise and strict definitions should be applied as they yield more reliable prevalence rates. Cultural and socio-economic factors contributing to the pattern of tobacco use among the elderly should be evaluated in future surveys. There is also a need of follow-up studies to prospectively evaluate tobacco use patterns among the elderly. In summary, our study is able to conclude that higher prevalence rates of tobacco use are found in males, although there is a possible trend for increasing tobacco use among elderly females, as observed in younger cohorts.

\section{Resumo}

O objetivo deste estudo foi combinar os resultados de pesquisas identificadas sobre a prevalência do tabagismo em idosos, para estimar sua prevalência mundial e possiveis fatores relacionados a este tipo de comportamento entre eles. A revisão da literatura incluiu busca nas bases de dados eletrônicas como MEDLINE LILACS e Biological Abstracts, busca manual em jornais especializados e nas referências citadas. A prevalência global combinada foi estimada usando-se o modelo de efeitos randômicos. O número total de idosos incluídos em todos os levantamentos foi 140.058, com dados disponiveis em todos os continentes. A prevalência de tabagismo foi de $13 \%$ em ambos os sexos (22\% homens e 8\% mulheres). As taxas de prevalência foram heterogêneas e estiveram associadas com a definição de tabagismo, aplicação do questionário e com a economia de cada país. A maior taxa de prevalência foi encontrada entre idosos do sexo masculino que vivem em países de renda mais alta.

Tabaco; Tabagismo; Idoso 


\section{Contributors}

V. Marinho reviewed the literature, selected the manuscripts, participated in the data analysis, and in the writing of the article. J. Laks participated in the selection of the manuscripts and in the writing of the article. E. S. F. Coutinho performed the statistical analyses and contributed to the discussion of the data in the manuscript. S. L. Blay designed the study and participated in all the steps, from the elaboration of the protocol to the final review of the manuscript.

\section{Conflicting interests}

Dr. Valeska Marinho has been working as a medical manager at GlaxoSmithKline Brazil.

\section{References}

1. World Health Organization. Confronting the tobacco epidemic in an era of trade liberation. Geneva: World Health Organization; 2003.

2. Centers for Disease Control and Prevention. Annual smoking-attributable mortality, years of potential life lost, and economic costs: United States, 1995-1999. MMWR Morb Mortal Wkly Rep 2002; 51:300-3.

3. Bratzler DW, Oehlert WH, Austelle A. Smoking in the elderly: it's never too late to quit. J Okla State Med Assoc 2002; 95:185-91.

4. Husten CG, Shelton DM, Chrismon JH, Lin YCW, Mowery P, Powell FA. Cigarette smoking and smoking cessation among older adults: United States, 1965-94. Tob Control 1997; 6:175-80.

5. Orleans CT, Jepson C, Resch N, Rimer BK. Quitting motives and barriers among older smokers: the 1986 adult use of tobacco survey revisited. Cancer 1994; 74:2055-61.

6. Jarvis MJ, Wardle J, Waller J, Owen L. Prevalence of hardcore smoking in England, and associated attitudes and beliefs: cross sectional study. BMJ 2003; 326:1061.

7. Townsend L, Flisher AJ, Gilreath T, King G. A systematic literature review of tobacco use among adults 15 years and older in sub-Saharan Africa. Drug Alcohol Depend 2006; 84:14-27.
8. Higgins JPT, Thompson SG, Deeks JJ, Altman DG Measuring inconsistency in meta-analyses. BMJ 2003; 327:557-60.

9. Clausen F, Sandberg E, Ingstad B, Hjortdahl P. Morbidity and health care utilizations among elderly people in Mmankgodi Village, Botswana. J Epidemiol Community Health 2000; 54:58-63.

10. Markides KS, Miller TQ, Ray LA. Changes in the smoking behavior of the elderly Mexican Americans in the southwest from 1982-1984 to 19931994. Prev Med 1999; 28:251-4.

11. Lima-Costa MF, Barreto SM, Uchôa E, Firmo JOA, Vidigal PG, Guerra HL. The Bambuí Health and Aging Study (BHAS): prevalence of risk factors and use of preventive health care services. Rev Panam Salud Pública 2001; 9:219-27.

12. Wiecha JM, Lee V, Hodgkins J. Patterns of smoking, risk factors for smoking, and smoking cessation among Vietnamese men in Massachusetts (United States). Tob Control 1998; 7:27-34.

13. Rani M, Bonu S, Jha P, Nguyen SN, Jamjoum L. Tobacco use in India: prevalence and predictors of smoking and chewing in a national cross sectional household survey. Tob Control 2003; 12:4.

14. Bursac Z, Campbell JE; Oklahoma Reach 2010 Steering Committee. Prevalence of current cigarette smoking among American Indians in Oklahoma: A comparison. J Okla State Med Assoc 2002; 95:155-8. 
15. Lau EM, Lee P, Lynn H, Sham A, Woo J. The epidemiology of cigarette smoking in Hong Kong Chinese women. Prev Med 2003; 37:383-8.

16. Gilliland FD, Mahler R, Davis SM. Non-ceremonial tobacco use among southwestern rural American Indians: the New Mexico American Indian Behavioral Risk Factor Survey. Tob Control 1998; 7 : 156-60.

17. Kamimoto LA, Easton AN, Maurice E, Husten CG, Macera CA. Surveillance for five health risks among older adults - United States, 1993-1997. MMWR CDC Surveill Summ 1999; 48:89-130.

18. Maxwell CJ, Hirdes JP. The prevalence of smoking and implications for quality of life among the community-based elderly. Am J Prev Med 1993; 9:338-45.

19. Shapo L, Gilmore AB, Coker R, McKee M, Shapo E. Prevalence and determinants of smoking in Tirana city, Albania: a population-based survey. Public Health 2003; 117:228-36.

20. Smedslund G, Ahn DK. Smoking initiation and cessation in Norway and the United States: a comparison of two cross-sectional surveys. J Subst Abuse 1998; 10:397-410.

21. Ganiwijaya T, Sjukrudin E, Backer GD, Suhana D, Brotoprawiro S, Sukandar H. Prevalence of cigarette smoking in a rural area of West Java, Indonesia. Tob Control 1995; 4:335-7.

22. Hamadeh RR, McPherson K, Doll R. Prevalence of smoking in Bahrain. Tob Control 1992; 1:102-6.

23. La Vecchia C, Pagano R, Decarli A, Ferraroni M. Smoking in Italy, 1990-1992. Tumori 1994; 80: 175-80.

24. Lima-Costa MF. A saúde dos adultos na região metropolitana de Belo Horizonte: um estudo epidemiológico de base populacional. Belo Horizonte: Núcleo de Estudos em Saúde Pública e Envelhecimento, Fundação Oswaldo Cruz/Universidade Federal de Minas Gerais; 2004.

25. Pagano R, La Vecchia C, Decarli A. Smoking in Italy, 1995. Tumori 1998; 84:456-9.

26. Shopland DR, Hartman AM, Gibson JT, Mueller MD, Kessler LG, Lynn WR. Cigarette smoking among U.S. adults by state and region: estimates from the current population survey. J Natl Cancer Inst 1996; 88:1748-58.

27. Balabanova D, Bobak M, McKee M. Patterns of smoking in Bulgaria. Tob Control 1998; 7:383-5.

28. Nebot M, Borrell C, Ballestin M, Villalbi JR. Prevalencia y características asociadas al consumo de tabaco en población general en Barcelona entre 1983 y 1992. Rev Clin Esp 1996; 196:359-64.

29. Osler M, Prescott E, Gottschau A, Bjerg A, Hein HO, Sjøl A, et al. Trends in smoking prevalence in Danish adults, 1964-1994. The influence of gender, age, and education. Scand J Soc Med 1998; 26:293-8.

30. Resnicow K, Futterman R, Weston RE, Royce J, Parms C, Freeman HP, et al. Smoking prevalence in Harlem, New York. Am J Health Promot 1996; 10:343-6.

31. Villalbí JR, Tomás Z, López MJ, Rodríguez M, Nebot M. La cambiante epidemiología del tabaquismo: Barcelona, 2000-2001. Rev Clin Esp 2004; 204:312-6.
32. Gutiérrez CM, Rioseco FC, Rojas AO, Casanova DZ, Cordero MR, Schiaffino MI. Prevalencia de tabaquismo en la población general de Valparaíso y Viña del Mar. Rev Med Chile 1995; 123:250-6.

33. Steyn K, Bradshaw D, Norman R, Laubscher R, Saloojee Y. Tobacco use in South Africans during 1998: the first demographic and health survey. J Cardiovasc Risk 2002; 9:161-70.

34. White V, Hill D, Siahpush M, Bobevski I. How has the prevalence of cigarette smoking changed among Australian adults? Trends in smoking prevalence between 1980 and 2001. Tob Control 2003; 12 Suppl 2:ii67-74.

35. Gilmore A, Pomerleau J, McKee M, Rose R, Haerpfer CW, Rotman D, et al. Prevalence of smoking in 8 countries of the former Soviet Union: results from the living conditions, lifestyles and health study. Am J Public Health 2004; 94:2177-87.

36. Jarallah JS, al-Rubeaan KA, al-Nuaim AR, al-Ruhaily AA, Kalantan KA. Prevalence and determinants of smoking in three regions of Saudi Arabia. Tob Control 1999; 8:53-6.

37. Lolio CA, Souza JMP, Santo AH, Buchalla CM. Prevalência de tabagismo em localidade urbana da região sudeste do Brasil. Rev Saúde Pública 1993; 27:262-5.

38. Cox HS, Williams JW, de Courten MP, Chitson P, Tuomilehto J, Zimmet PZ. Decreasing prevalence of cigarette smoking in the middle income country of Mauritius: questionnaire survey. BMJ 2000; 321:345-9.

39. McKee M, Bobak M, Rose R, Shkolnikov V, Chenet L, Leon D. Patterns of smoking in Russia. Tob Control 1998; 7:22-6.

40. Yang G, Fan L, Tan J, Qi G, Zhang Y, Samet JM, et al. Smoking in China: findings of the 1996 National Prevalence Survey. JAMA 1999; 282:1247-53.

41. Hu TW, Tsai YW. Cigarette consumption in rural China: survey results from 3 provinces. Am J Public Health 2000; 90:1785-7.

42. Ohida T, Kamal AM, Takemura S, Sone T, Mochizuki Y, Kawaminami K. Relation between smoking prevalence and various social factors in Japan. Keio J Med 2001; 50:263-8.

43. Gong YL, Koplan JP, Feng W, Chen CH, Zheng P, Harris JR. Cigarette smoking in China. Prevalence, characteristics, and attitudes in Minhang District. JAMA 1995; 274:1232-4.

44. Nasir K, Rehan N. Epidemiology of cigarette smoking in Pakistan. Addiction 2001; 96:1847-54.

45. Shah SM, Arif AA, Delclos GL, Khan AR, Khan A. Prevalence and correlates of smoking on the roof of the world. Tob Control 2001; 10:e1.

46. Woollery T, Trosclair A, Husten C, Caraballo RC, Kahende J. Cigarette smoking among adults: United States, 2001. MMWR Morb Mortal Wkly Rep 2003; 52:953-4.

47. Instituto Nacional de Câncer. Inquérito domiciliar sobre comportamentos de risco e morbidade referida de doenças e agravos não transmissíveis. Brasil, 15 capitais e Distrito Federal 2002-2003. Rio de Janeiro: Instituto Nacional de Câncer; 2004.

48. Gilmore AB, McKee M, Telishevska M, Rose R. Epidemiology of smoking in Ukraine, 2000. Prev Med 2001; 33:453-61. 
49. Woodward A, Newland H, Kinahoi M. Smoking in the Kingdom of Tonga: a report from a national survey. Tob Control 1994; 3:41-5.

50. Haidinger G, Waldhoer T, Vutuc C. The prevalence of smoking in Austria. Prev Med 1998; 27:50-5.

51. Santos AC, Barros H. Smoking patterns in a community sample of Portuguese adults, 1999-2000. Prev Med 2004; 38:114-9.

52. Arnett DK, Sprafka JM, McGovern PG, Jacobs Jr. DR, Shahar E, McCarty M, et al. Trends in cigarette smoking: the Minnesota Heart Survey, 1980 through 1992. Am J Public Health 1998; 88:1230-3.

53. Gilmore AB, McKee M, Rose R. Prevalence and determinants of smoking in Belarus: a national household survey, 2000. Eur J Epidemiol 2001; 17:245-53.

54. Hill DJ, White VM, Scollo MM. Smoking behaviors of Australian adults in 1995: trends and concerns. Med J Aust 1998; 168:209-13.

55. Idris AM, Ibrahim YE, Warnakulasuriya KA, Cooper DJ, Johnson NW, Nilsen R. Toombak use and cigarette smoking in the Sudan: estimates of prevalence in the Nile state. Prev Med 1998; 27:597-603.

56. Centers for Disease Control and Prevention. Cigarette smoking among adults: United States, 1992, and changes in the definition of current cigarette smoking. MMWR Morb Mortal Wkly Rep 1994; 43:342-6.

57. Grunberg NE, Winders SE, Wewers ME. Gender differences in tobacco use. Health Psychol 1991; 10:143-53

58. Global Youth Tobacco Survey Collaborating Group. Differences in worldwide tobacco use by gender: findings from the Global Youth Tobacco Survey. J Sch Health 2003; 73:207-15.

59. Arday DR, Tomar SL, Nelson DE, Merritt RK, Schooley MW, Mowery P. State smoking prevalence estimates: a comparison of the Behavioral Risk Factor Surveillance System and current population surveys. Am J Public Health 1997; 87:1665-9.
60. Rissel C, Ward JE, Jorm L. Estimates of smoking and related behaviour in an immigrant Lebanese community: does survey method matter? Aust N Z J Public Health 1999; 23:534-7.

61. Starr GJ, Dal Grande E, Taylor AW, Wilson DH. Reliability of self-reported behavioural health risk factors in a South Australian telephone survey. Aust N Z J Public Health 1999; 23:528-30.

62. Donovan RJ, Holman CD, Corti B, Jalleh G. Faceto-face household interviews versus telephone interviews for health surveys. Aust N Z J Public Health 1997; 21:134-40.

63. Solberg LI, Hollis JA, Stevens VJ, Rigotti NA, Quinn VP, Aickin M. Does methodology affect the ability to monitor tobacco control activities? Implications for HEDIS and other performance measures. Prev Med 2003; 37:33-40.

64. Selikoff IJ, Seidman H. Evaluation of selection bias in a cross-sectional survey. Am J Ind Med 1991; 20:615-27.

65. Patrick DL, Cheadle A, Thompson DC, Diehr P, Koepsell T, Kinne S. The validity of self-reported smoking: a review and meta-analysis. Am J Public Health 1994; 84:1086-93

66. Subramanian SV, Nandy S, Kelly M, Gordon D, Davey Smith G. Patterns and distribution of tobacco consumption in India: cross sectional multilevel evidence from the 1998-9 national family health survey. BMJ 2004; 328:801-6.

67. Siahpush M. Socioeconomic status and tobacco expenditure among Australian households: results from the 1998-99 Household Expenditure Survey. J Epidemiol Community Health 2003; 57:798-801.

Submitted on $24 / \mathrm{Feb} / 2010$

Final version resubmitted on 14/Jun/2010

Approved on 29/Jun/2010 